\title{
Estimates of Dimension Reduction Errors for Stationary Reaction-Diffusion Problems
}

\author{
S. Repin * T. Samrowski ${ }^{\dagger}$
}

\begin{abstract}
We consider the stationary reaction-diffusion model in a domain $\Omega \in \mathbb{R}^{n}$ having the size along one coordinate direction essentially smaller than along the others. By an energy type argumentation we deduce two simplified models of lower dimension (zero-, and first-order models), which are defined on a domain of the dimension $n-1$. For these models, we derive fully computable estimates of the difference between the solution of the original problem and $n$-dimensional reconstructions generated by solutions of dimensionally reduced problems.
\end{abstract}

2000 Mathematics Subject Classification: 35J20, 65N15, 65N30

Key words and phrases: modeling error, dimension reduction, thin domain, a posteriori error estimate, reliability

\section{Introduction}

In the past decades, model reduction has become an essential tool in analysis and simulation of many complex physical models. It is often beneficial to reduce the dimension of the data in order to reduce the computation cost or even improve the efficiency and accuracy of the data analysis. The method of dimensional reduction is a typical way of solving boundary value problems associated with domains, where the size along one coordinate direction is much smaller than the others. In such a case, the solution $u^{(n)}$ of a $n$-dimensional problem $\mathcal{P}^{(n)}$ (let us assume that $u^{(n)}$ belongs to a Banach space $V)$ is approximated by a solution $u^{(n-k)}$ of a certain simplified problem $\mathcal{P}^{(n-k)}$, where $k$ is a positive integer number. A computable approximation $u_{\tau}^{(n-k)}$ of $u^{(n-k)}$, which present a reliable information on $u^{(n)}$, can be obtained, e.g., by projecting $\mathcal{P}^{(n-k)}$ onto a finite dimensional space and solving the corresponding discrete problem $\mathcal{P}_{\tau}^{(n-k)}$ (for the detailed discussion on the hierarchy of reduced problems see, e.g., [4], [18]).

Due to the fact that the functions $u^{(n)}, u^{(n-k)}$, and $u_{\tau}^{(n-k)}$ belong to different spaces, a dimension reconstruction operator $\mathfrak{K}: V^{(n-k)} \rightarrow V$, which forms images of solutions of

${ }^{*}$ V.A. Steklov Institute of Mathematics, Fontanka 27, 191011 St. Petersburg, Russia; E-mail: repin@pdmi.ras.ru

${ }^{\dagger}$ Institute of Mathematics, Zurich University, Winterthurerstrasse 190, CH-8057 Zurich, Switzerland; E-mail: tatiana.samrowski@math.uzh.ch 
$(n-k)$-dimensional problems in the $n$-dimensional space $V$, should be introduced. We assume that $\mathfrak{K}$ satisfies (except the obvious conditions of computational simplicity and boundedness) the Lipschitz condition

$$
\left\|\mathfrak{K} v_{1}-\mathfrak{K} v_{2}\right\|_{V} \leq \mathrm{C}_{\mathfrak{K}}\left\|v_{1}-v_{2}\right\|_{V^{(n-k)}} \quad \text { for all } \quad v_{1}, v_{2} \in V^{(n-k)},
$$

where the constant $\mathrm{C}_{\mathfrak{K}}>0$ does not depend on $v_{1}$ and $v_{2}$.

In order to determine the difference between $u^{(n)}$ and the function $\mathfrak{K} u_{\tau}^{(n-k)}$, we obtain by the triangle inequality that

$$
\begin{aligned}
\left\|u^{(n)}-\mathfrak{K} u_{\tau}^{(n-k)}\right\|_{V} & \leq\left\|u^{(n)}-\mathfrak{K} u^{(n-k)}\right\|_{V}+\left\|\mathfrak{K} u^{(n-k)}-\mathfrak{K} u_{\tau}^{(n-k)}\right\|_{V} \\
& \stackrel{(1.1)}{\leq}\left\|u^{(n)}-\mathfrak{K} u^{(n-k)}\right\|_{V}+\mathrm{C}_{\mathfrak{K}}\left\|u^{(n-k)}-u_{\tau}^{(n-k)}\right\|_{V^{(n-k)}}
\end{aligned}
$$

The first term in (1.2) represents the modeling error due to the dimension reduction, the second one is related to the numerical discretization. The additive splitting gives insights how these two parts of the total error are balanced.

Historically, the subject of error estimation in dimension reduction models was mainly focused on a priori asymptotic error estimates that evaluate the difference between original and reduced models in terms of small (geometric) parameters. In particular, such type estimates has been properly investigated for diffusion problems and in elasticity theory (cf. $[1,5,6])$.

Estimates of the different type that can be used to evaluate modeling errors for problems with a given thickness were derived in [15] for diffusion problems and in [12] for stressed planes.

In this paper, we present computable estimates of the modeling error $\left\|u^{(n)}-\mathfrak{K} u^{(n-k)}\right\|_{V}$ in the case of the approximation of the three-dimensional stationary reaction diffusion problem by the two-dimensional model problem.

The paper is organized as follows. In Section 2, we specify the original problem and introduce the notation. Then, in Section 3, we present and discuss two different dimension reduction models: The so called zero-order and first-order reduced model. In Section 4 , the main theorems, which provide guaranteed and fully computable upper bounds of dimension reduction errors for the both reduced models, are given and proved. Particular cases of dimension geometry are surveyed in Section 5 .

\section{Statement of the problem and notation}

We consider a simply connected and bounded domain

$$
\Omega:=\left\{x \in \mathbb{R}^{3} \mid\left(x_{1}, x_{2}\right) \in \widehat{\Omega}, d_{\ominus}\left(x_{1}, x_{2}\right)<x_{3}<d_{\oplus}\left(x_{1}, x_{2}\right)\right\} \in \mathbb{R}^{3},
$$

where $\widehat{\Omega} \subset \mathbb{R}^{2}$ denotes the orthogonal projection of $\Omega$ on the $\left(x_{1}, x_{2}\right)$-plane (see Figure 1) and $d_{\ominus}$ and $d_{\oplus}$ are Lipschitz continuous and differentiable functions of $\left(x_{1}, x_{2}\right)$ - 
coordinates. We denote the lower and upper faces of $\Omega$ by

$$
\Gamma_{\ominus}:=\left\{x \in \mathbb{R}^{3} \mid\left(x_{1}, x_{2}\right) \in \widehat{\Omega}, x_{3}=d_{\ominus}\left(x_{1}, x_{2}\right)\right\}
$$

and

$$
\Gamma_{\oplus}:=\left\{x \in \mathbb{R}^{3} \mid\left(x_{1}, x_{2}\right) \in \widehat{\Omega}, x_{3}=d_{\oplus}\left(x_{1}, x_{2}\right)\right\},
$$

respectively, and the lateral boundaries associated with Robin and Dirichlet boundary conditions by

$$
\Gamma_{R}:=\left\{x \in \mathbb{R}^{3} \mid\left(x_{1}, x_{2}\right) \in \widehat{\Gamma}_{R}, d_{\ominus}\left(x_{1}, x_{2}\right)<x_{3}<d_{\oplus}\left(x_{1}, x_{2}\right)\right\}
$$

and

$$
\Gamma_{D}:=\left\{x \in \mathbb{R}^{3} \mid\left(x_{1}, x_{2}\right) \in \widehat{\Gamma}_{D}, d_{\ominus}\left(x_{1}, x_{2}\right)<x_{3}<d_{\oplus}\left(x_{1}, x_{2}\right)\right\}
$$
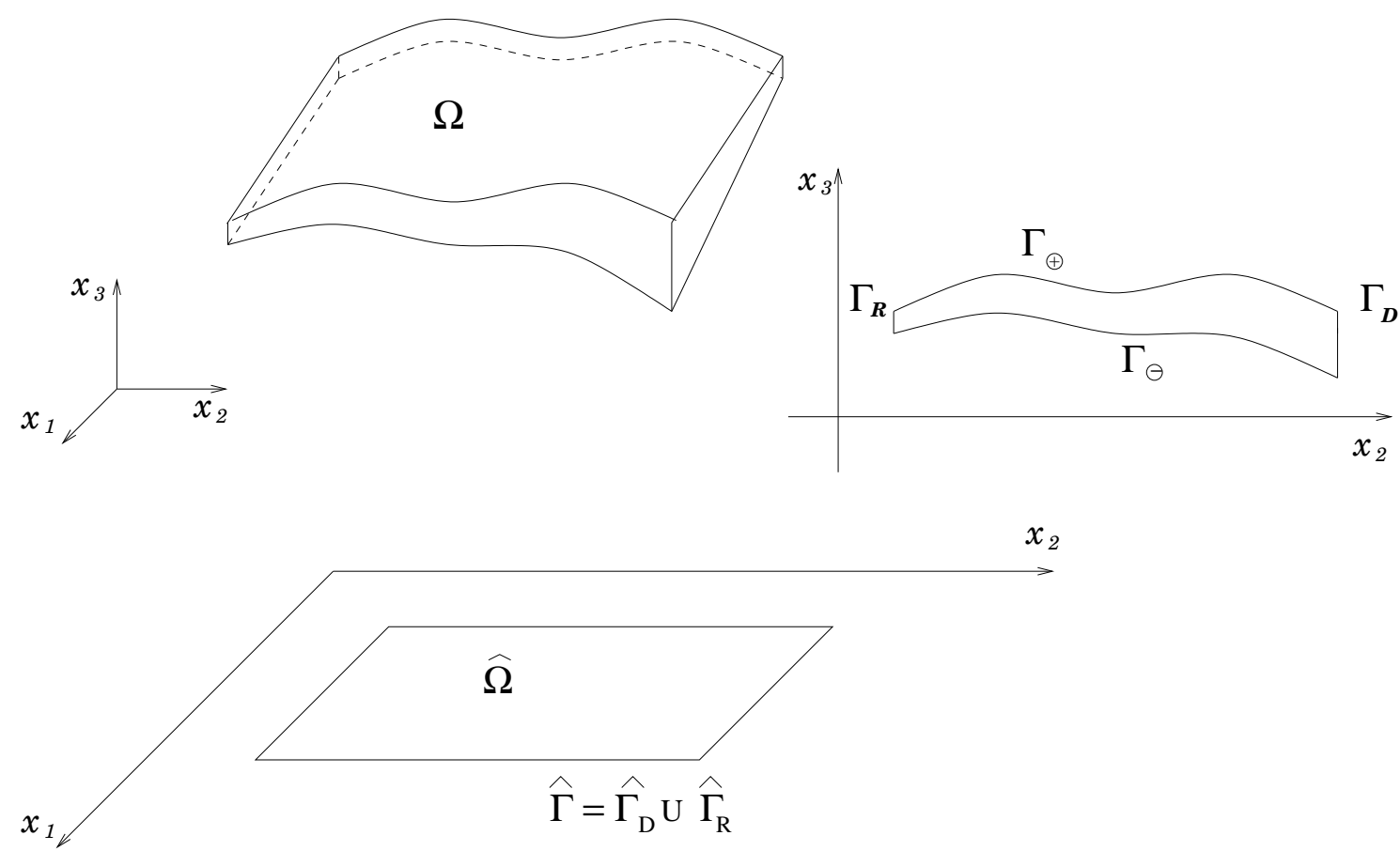

Figure 1: Domain geometry and notation.

In $\Omega$, we consider the stationary reaction-diffusion problem with mixed Dirichlet-Robin 
boundary conditions (which we call Problem $\mathcal{P}$ )

$$
\begin{aligned}
-\operatorname{Div}(\mathbf{A}(x) \nabla u)+\rho^{2}(x) u & =f & & \text { in } \Omega, \\
\kappa(x) u+\mathbf{A}(x) \nabla u \cdot \boldsymbol{\nu}_{R} & =0 & & \text { on } \Gamma_{R}, \\
\mathbf{A}(x) \nabla u \cdot \boldsymbol{\nu}_{\ominus} & =F_{\ominus} & & \text { on } \Gamma_{\ominus}, \\
\mathbf{A}(x) \nabla u \cdot \boldsymbol{\nu}_{\oplus} & =F_{\oplus} & & \text { on } \Gamma_{\oplus}, \\
u & =0 & & \text { on } \Gamma_{D},
\end{aligned}
$$

where

$$
f \in L^{2}(\Omega), F_{\ominus} \in L^{2}\left(\Gamma_{\ominus}\right), F_{\oplus} \in L^{2}\left(\Gamma_{\oplus}\right), \rho^{2}(x) \in L^{\infty}(\Omega), 0 \leq \kappa(x) \not \equiv 0,
$$

$\boldsymbol{\nu}_{R}, \boldsymbol{\nu}_{\ominus}$ and $\boldsymbol{\nu}_{\oplus}$ are outward normal vectors at $\Gamma_{R}, \Gamma_{\ominus}$ and $\Gamma_{\oplus}$, respectively, and $\kappa(x)$ and $\rho^{2}(x)$ are some known real functions. We assume that the matrix

$$
\mathbf{A}(x)=\left(a_{i j}(x)\right)_{i, j=\overline{1,3}}: \Omega \rightarrow \mathbb{R}_{\mathrm{sym}}^{3 \times 3}
$$

has the components from $L_{\infty}(\Omega)$ and is uniformly positive definite, i.e., there exist constants $0<c_{1}<c_{2}<\infty$ such that

$$
c_{1}|\xi|^{2} \leq \mathbf{A}(x) \xi \cdot \xi \leq c_{2}|\xi|^{2} \quad \text { for all } \xi \in \mathbb{R}^{3} \text { a. e. in } \Omega,
$$

and set

$$
\mathbf{A}_{p, 0}(x):=\left(a_{i j}(x)\right)_{i, j=\overline{1,2}}: \Omega \rightarrow \mathbb{R}_{\mathrm{sym}}^{2 \times 2} .
$$

It is well known that the generalized solution $u$ of $(2.2)$ is a function in

$$
V_{0}:=\left\{v \in H^{1}(\Omega) \mid v=0 \text { on } \Gamma_{D}\right\}
$$

that satisfies the following integral relation for all $w \in V_{0}$

$$
\begin{aligned}
\int_{\Omega}\left(\mathbf{A}(x) \nabla u \cdot \nabla w d x+\rho^{2}(x) u w\right)+\int_{\Gamma_{R}} & \kappa(x) u w d s \\
& =\int_{\Omega} f w d x+\int_{\Gamma_{\ominus}} F_{\ominus} w d s+\int_{\Gamma_{\oplus}} F_{\oplus} w d s .
\end{aligned}
$$

It exists, is unique, and continuously depends on the data with respect to the norm of $H^{1}(\Omega)$.

From now on, we assume that

$$
\operatorname{diam} \widehat{\Omega} \gg \max _{\left(x_{1}, x_{2}\right) \in \bar{\Omega}} d\left(x_{1}, x_{2}\right)
$$

where

$$
d\left(x_{1}, x_{2}\right)=d_{\oplus}\left(x_{1}, x_{2}\right)-d_{\ominus}\left(x_{1}, x_{2}\right),
$$


is the domain thickness in point $\left(x_{1}, x_{2}\right)$ and $d\left(x_{1}, x_{2}\right) \geq d_{*}>0$ for all $\left(x_{1}, x_{2}\right) \in \overline{\widehat{\Omega}}$.

We will use the notation $\widehat{x}:=\left(x_{1}, x_{2}\right), \widehat{x} \in \widehat{\Omega}$ and mark all functions depending only on $\left(x_{1}, x_{2}\right)$ by $\widehat{~}$. We also will distinguish between the 3 - and 2-dimensional divergence operator:

$$
\operatorname{Div} \boldsymbol{\tau}=\frac{\partial \boldsymbol{\tau}_{1}}{\partial x_{1}}+\frac{\partial \boldsymbol{\tau}_{2}}{\partial x_{2}}+\frac{\partial \boldsymbol{\tau}_{3}}{\partial x_{3}}, \quad \operatorname{div} \widehat{\boldsymbol{\tau}}=\frac{\partial \widehat{\boldsymbol{\tau}}_{1}}{\partial x_{1}}+\frac{\partial \widehat{\boldsymbol{\tau}}_{2}}{\partial x_{2}},
$$

and the 3- and 2-dimensional gradient:

$$
\nabla u:=\left(\frac{\partial u}{\partial x_{1}}, \frac{\partial u}{\partial x_{2}}, \frac{\partial u}{\partial x_{3}}\right), \quad \widehat{\nabla} \widehat{u}:=\left(\frac{\partial \widehat{u}}{\partial x_{1}}, \frac{\partial \widehat{u}}{\partial x_{2}}\right) .
$$

\section{Dimensionally reduced models}

In this section, we consider two different models of the dimension reduction. The first one (zero-order reduced model) is based on the hypothesis that "the exact solution is almost constant with respect to the $x_{3}$-coordinate" .

According to this hypothesis, we expect that the exact solution $u$ may be well-approximated by the functions from the subspace

$$
\begin{aligned}
V_{0}^{(0)}:=\left\{v \in V_{0} \mid \exists \widehat{v} \in H^{1}(\widehat{\Omega}) \text { such that } \widehat{v}\right. & =0 \text { on } \widehat{\Gamma}_{D} \text { and } \\
v(x) & \left.=\widehat{v}(\widehat{x}) \text { for a.e. } x=\left(\widehat{x}, x_{3}\right) \in \Omega\right\} .
\end{aligned}
$$

Then, the energy-norm projection of $u$ onto the subspace $V_{0}^{(0)}$ yields the reduced

$\underline{\text { Problem } \widehat{\mathcal{P}}^{(0)}}$ : Find $u^{(0)} \in V_{0}^{(0)}$ such that for all $w \in V_{0}^{(0)}$

$$
\int_{\Omega}\left(\mathbf{A} \nabla u^{(0)} \cdot \nabla w+\rho^{2} u^{(0)} w\right)+\int_{\Gamma_{R}} \kappa u^{(0)} w=\int_{\Omega} f w+\int_{\Gamma_{\ominus}} F_{\ominus} w+\int_{\Gamma_{\oplus}} F_{\oplus} w .
$$

The difference

$$
e:=u-u^{(0)}
$$

is the modeling error generated by the zero-order model. We note that in this simplest model the function $u^{(0)}$ is plane, so that we can denote it by $\widehat{u}^{(0)}$. Certainly, the problem (3.2) must be mathematically correct (i.e., possess a solution which continuously depends on the problem data). To show this, we integrate (3.2) over the direction $x_{3}$. Let the operation $\left({ }^{\sim}\right)$ denotes the averaging with respect to $x_{3}$, e.g., for all $g \in L_{1}(\Omega)$

$$
\widetilde{g}_{i}(\widehat{x}):=\frac{1}{d(\widehat{x})} \int_{d_{\ominus}(\widehat{x})}^{d_{\oplus}(\widehat{x})} x_{3}^{i} g\left(\widehat{x}, x_{3}\right) d x_{3} \text { for a.e. } \widehat{x} \in \widehat{\Omega}, i=0,1,2 .
$$

Having noticed that

$$
\int_{\Gamma_{\ominus, \oplus}} F_{\ominus, \oplus} \widehat{w}=\int_{\widehat{\Omega}} \widehat{F}_{\ominus, \oplus}(\widehat{x}) \widehat{w}(\widehat{x}) \sqrt{1+\left|\nabla d_{\ominus, \oplus}(\widehat{x})\right|^{2}}
$$


and defined for $k=0,1,2$

$$
\widetilde{\mathbf{A}}_{p, k}(\widehat{x}):=\frac{1}{d(\widehat{x})} \int_{d_{\ominus}(\widehat{x})}^{d_{\oplus}(\widehat{x})} x_{3}^{k} \mathbf{A}_{p, 0}(x) d x_{3} \quad \text { with } \quad \widetilde{\mathbf{A}}_{p, k}(\widehat{x})=\left(\widetilde{a}_{i j}(\widehat{x})\right)_{i, j=\overline{1,2}}: \widehat{\Omega} \rightarrow \mathbb{R}_{s y m}^{2 \times 2},
$$

as well as

$$
\widehat{f}_{i}(\widehat{x}):=\widetilde{f}_{i}(\widehat{x})+\frac{1}{d(\widehat{x})}\left(\widehat{F}_{\ominus}(\widehat{x}) \sqrt{1+\left|\nabla d_{\ominus}(\widehat{x})\right|^{2}}+\widehat{F}_{\oplus}(\widehat{x}) \sqrt{1+\left|\nabla d_{\oplus}(\widehat{x})\right|^{2}}\right),
$$

we can rewrite $(3.2)$ as follows: Find $\widehat{u}^{(0)} \in V_{0}^{(0)}$ such that for all $\widehat{w} \in V_{0}^{(0)}$

$$
\int_{\widehat{\Omega}} d(\widehat{x})\left(\widetilde{\mathbf{A}}_{p, 0}(\widehat{x}) \widehat{\nabla} \widehat{u}^{(0)} \cdot \widehat{\nabla} \widehat{w}+\widetilde{\rho}_{0}^{2}(\widehat{x}) \widehat{u}^{(0)} \widehat{w}\right) d \widehat{x}+\int_{\widehat{\Gamma}_{R}} d(\widehat{x}) \widetilde{\kappa}_{0} \widehat{u}^{(0)} \widehat{w} d \widehat{s}=\int_{\widehat{\Omega}} d(\widehat{x}) \widehat{f_{0}}(\widehat{x}) \widehat{w} d \widehat{x} .
$$

Problem (3.6) is the week form of a two-dimensional reaction-diffusion problem with the homogeneous boundary conditions:

$$
\begin{aligned}
-\operatorname{div}\left(d(\widehat{x}) \widetilde{\mathbf{A}}_{p, 0}(\widehat{x}) \widehat{\nabla} \widehat{u}^{(0)}\right)+d(\widehat{x}) \widetilde{\rho}_{0}^{2}(\widehat{x}) \widehat{u}^{(0)} & =d(\widehat{x}) \widehat{f_{0}}(\widehat{x}) & & \text { in } \widehat{\Omega}, \\
\widetilde{\kappa}_{0}(\widehat{x}) \widehat{u}^{(0)}+\widetilde{\mathbf{A}}_{p, 0}(\widehat{x}) \widehat{\nabla} \widehat{u}^{(0)} \cdot \widehat{\boldsymbol{\nu}}_{R} & =0 & & \text { on } \widehat{\Gamma}_{R}, \\
\widehat{u}^{(0)} & =0 & & \text { on } \widehat{\Gamma}_{D} .
\end{aligned}
$$

In order to derive a more sophisticated reduced problem (which we hereafter call firstorder reduced model), we accept the hypothesis "the exact solution $u$ is almost linear with respect to the $x_{3}$-coordinate", and expect that the solution $u$ may be well approximated by the functions from the subspace

$$
\begin{aligned}
& V_{0}^{(1)}:=\left\{v \in V_{0} \mid \exists \widehat{v}_{1}, \widehat{v}_{2} \in H^{1}(\widehat{\Omega}) \text { such that } \widehat{v}_{1}=\widehat{v}_{2}=0 \text { on } \widehat{\Gamma}_{D}\right. \text { and } \\
& \left.\qquad v(x)=\widehat{v}_{1}(\widehat{x})+x_{3} \widehat{v}_{2}(\widehat{x}) \text { for a.e. } x=\left(\widehat{x}, x_{3}\right) \in \Omega\right\} .
\end{aligned}
$$

To derive the corresponding reduced problem, we should minimize the functional

$$
J(v):=\frac{1}{2} \int_{\Omega} \mathbf{A} \nabla v \cdot \nabla v d s+\frac{1}{2} \int_{\Gamma_{R}} \kappa v^{2} d s-\int_{\Omega} f v d x .
$$

It is clear that $J(v)$ is convex and coercive in the space $V_{0}^{(1)}$. Then by the standard arguments of convex analysis there exists a unique minimizer

$$
u^{(1)}=\underset{v \in V_{0}^{(1)}}{\arg \min } J(v) .
$$

Hence, for all $v:=u^{(1)}+\alpha w$ with $u^{(1)}$ from (3.9), and any $w \in V_{0}^{(1)}, \alpha \in \mathbb{R}$, holds the inequality $J(v) \geq J(u)$. Due to the definition of the first variation, we have

$$
\partial J(u, w)=\left.\frac{d}{d \alpha} J(u+\alpha w)\right|_{\alpha=0}=: T_{1}+T_{2}+T_{3}-T_{4}
$$


and obtain for the first term of (3.10) by setting $u^{(1)}=\widehat{u}_{1}^{(1)}+x_{3} \widehat{u}_{2}^{(1)}$ and $w=\widehat{w}_{1}+x_{3} \widehat{w}_{2}$

$$
\begin{aligned}
T_{1}:= & \frac{d}{d \alpha}\left[\int_{\Omega} \frac{1}{2} \mathbf{A}\left(\nabla\left(\widehat{u}_{1}^{(1)}+\alpha \widehat{w}_{1}+x_{3} \widehat{u}_{2}^{(1)}+x_{3} \alpha \widehat{w}_{2}\right)\right) \cdot\left(\nabla\left(\widehat{u}_{1}^{(1)}+\alpha \widehat{w}_{1}+x_{3} \widehat{u}_{2}^{(1)}+x_{3} \alpha \widehat{w}_{2}\right)\right) d x\right. \\
& -\left.\int_{\Omega} \frac{1}{2} \mathbf{A}\left(\nabla\left(\widehat{u}_{1}^{(1)}+x_{3} \widehat{u}_{2}^{(1)}\right) \cdot\left(\nabla\left(\widehat{u}_{1}^{(1)}+x_{3} \widehat{u}_{2}^{(1)}\right)\right) d x\right]\right|_{\alpha=0} \\
= & \int_{\Omega}\left(\mathbf{A} \nabla\left(\widehat{u}_{1}^{(1)}+x_{3} \widehat{u}_{2}^{(1)}\right) \cdot \nabla \widehat{w}_{1}+x_{3} \mathbf{A} \nabla\left(\widehat{u}_{1}^{(1)}+2 x_{3} \widehat{u}_{2}^{(1)}\right) \cdot \nabla \widehat{w}_{2}\right) d x .
\end{aligned}
$$

By similar computations, we find that

$$
\begin{gathered}
T_{2}:=\int_{\Omega}\left(\rho^{2}\left(\widehat{u}_{1}^{(1)}+x_{3} \widehat{u}_{2}^{(1)}\right) \widehat{w}_{1}+x_{3} \rho^{2}\left(\widehat{u}_{1}^{(1)}+x_{3} \widehat{u}_{2}^{(1)}\right) \widehat{w}_{2}\right) d x, \\
T_{3}:=\int_{\Gamma_{R}}\left(\kappa\left(\widehat{u}_{1}^{(1)}+x_{3} \widehat{u}_{2}^{(1)}\right) \widehat{w}_{1}+x_{3} \kappa\left(\widehat{u}_{1}^{(1)}+x_{3} \widehat{u}_{2}^{(1)}\right) \widehat{w}_{2}\right) d s,
\end{gathered}
$$

and

$$
T_{4}:=\int_{\Omega} f\left(\widehat{w}_{1}+x_{3} \widehat{w}_{2}\right) d x .
$$

Hence, the energy-norm projection of $u$ onto the subspace $V_{0}^{(1)}$ yields (with definitions (3.3), (3.4) and (3.5)) the following

Problem $\widehat{\mathcal{P}}^{(1)}$ : Find $\widehat{u}_{1}^{(1)}, \widehat{u}_{2}^{(1)} \in H^{1}(\widehat{\Omega})$ with $\widehat{u}_{1}^{(1)}=\widehat{u}_{2}^{(1)}=0$ on $\widehat{\Gamma}_{D}$ such that for all $\widehat{w}_{1}, \widehat{w}_{2} \in H^{1}(\widehat{\Omega})$ with $\widehat{w}_{1}=\widehat{w}_{2}=0$ on $\widehat{\Gamma}_{D}$ the following relations hold:

$$
\begin{aligned}
& \int_{\widehat{\Omega}} d(\widehat{x})(\left(\widetilde{\mathbf{A}}_{p, 0}(\widehat{x}) \hat{\nabla} \widehat{u}_{1}^{(1)}+\widetilde{\mathbf{A}}_{p, 1}(\widehat{x}) \widehat{\nabla} \widehat{u}_{2}^{(1)}\right) \cdot \widehat{\nabla} \widehat{w}_{1} \\
&\left.\left.\left.\left.\left.+\int_{\widehat{\Gamma}_{R}} d(\widehat{x})\left(\widetilde{\kappa}_{0}(\widehat{x}) \widehat{u}_{1}^{(1)}+\widetilde{\kappa}_{1}(\widehat{x}) \widehat{u}_{2}^{(1)}\right)\right) \widehat{w}_{1} d \widehat{s}=\int_{\widehat{\Omega}} d(\widehat{x}) \widehat{u}_{1}^{(1)}+\widetilde{\rho}_{1}^{2}(\widehat{x}) \widehat{u}_{0}^{(1)}\right)\right) \widehat{w}_{1}\right) d \widehat{x}\right) \widehat{w}_{1} d \widehat{x}, \\
&\left.\int_{\widehat{\Omega}} d(\widehat{x})\left(\left(\widetilde{\mathbf{A}}_{p, 1}(\widehat{x}) \widehat{\nabla} \widehat{u}_{1}^{(1)}+\widetilde{\mathbf{A}}_{p, 2}(\widehat{x}) \widehat{\nabla} \widehat{u}_{2}^{(1)}\right) \cdot \widehat{\nabla} \widehat{w}_{2}+\left(\widetilde{\rho}_{1}^{2}(\widehat{x}) \widehat{u}_{1}^{(1)}+\widetilde{\rho}_{2}^{2}(\widehat{x}) \widehat{u}_{2}^{(1)}\right)\right) \widehat{w}_{2}\right) d \widehat{x} \\
&\left.+\int_{\widehat{\Gamma}_{R}} d(\widehat{x})\left(\widetilde{\kappa}_{1}(\widehat{x}) \widehat{u}_{1}^{(1)}+\widetilde{\kappa}_{2}(\widehat{x}) \widehat{u}_{2}^{(1)}\right)\right) \widehat{w}_{2} d \widehat{s}=\int_{\widehat{\Omega}} d(\widehat{x}) \widehat{f}_{1}(\widehat{x}) \widehat{w}_{2} d \widehat{x} .
\end{aligned}
$$


It is clear that (3.11) is the week form of a system of two two-dimensional reactiondiffusion problems with the homogeneous boundary condition:

$$
\begin{aligned}
-\operatorname{div} d(\widehat{x})\left(\widetilde{\mathbf{A}}_{p, 0}(\widehat{x}) \widehat{\nabla} \widehat{u}_{1}^{(1)}+\widetilde{\mathbf{A}}_{p, 1}(\widehat{x}) \widehat{\nabla} \widehat{u}_{2}^{(1)}\right)+d(\widehat{x})\left(\widetilde{\rho}_{0}^{2}(\widehat{x}) \widehat{u}_{1}^{(1)}+\widetilde{\rho}_{1}^{2}(\widehat{x}) \widehat{u}_{2}^{(1)}\right)=d(\widehat{x}) \widehat{f}_{0}(\widehat{x}) & \text { in } \widehat{\Omega}, \\
-\operatorname{div} d(\widehat{x})\left(\widetilde{\mathbf{A}}_{p, 1}(\widehat{x}) \widehat{\nabla} \widehat{u}_{1}^{(1)}+\widetilde{\mathbf{A}}_{p, 2}(\widehat{x}) \widehat{\nabla} \widehat{u}_{2}^{(1)}\right)+d(\widehat{x})\left(\widetilde{\rho}_{1}^{2}(\widehat{x}) \widehat{u}_{1}^{(1)}+\widetilde{\rho}_{2}^{2}(\widehat{x}) \widehat{u}_{2}^{(1)}\right)=d(\widehat{x}) \widehat{f}_{1}(\widehat{x}) & \text { in } \widehat{\Omega}, \\
\widetilde{\kappa}_{0}(x) \widehat{u}_{1}^{(1)}+\widetilde{\kappa}_{1}(x) \widehat{u}_{2}^{(1)}+\left(\widetilde{\mathbf{A}}_{p, 0}(\widehat{x}) \widehat{\nabla} \widehat{u}_{1}^{(1)}+\widetilde{\mathbf{A}}_{p, 1}(\widehat{x}) \widehat{\nabla} \widehat{u}_{2}^{(1)}\right) \cdot \widehat{\boldsymbol{\nu} R}=0 & \text { on } \widehat{\Gamma}_{R}, \\
\widetilde{\kappa}_{1}(x) \widehat{u}_{1}^{(1)}+\widetilde{\kappa}_{2}(x) \widehat{u}_{2}^{(1)}+\left(\widetilde{\mathbf{A}}_{p, 1}(\widehat{x}) \widehat{\nabla} \widehat{u}_{1}^{(1)}+\widetilde{\mathbf{A}}_{p, 2}(\widehat{x}) \widehat{\nabla} \widehat{u}_{2}^{(1)}\right) \cdot \widehat{\boldsymbol{\nu} R}=0 & \text { on } \widehat{\Gamma}_{R}, \\
\widehat{u}_{1}^{(1)}=\widehat{u}_{2}^{(1)}=0 & \text { on } \widehat{\Gamma}_{D} .
\end{aligned}
$$

Remark 3.1 We note that if the domain is bounded by plane parallel faces and the coefficients $a_{i j}(x)$ and $\kappa(x)$ are symmetric functions with respect to $x_{3}$, the system (3.12) is decomposed into two independent reaction-diffusion problems with the homogeneous boundary conditions:

$$
\begin{array}{rlrl}
-\operatorname{div} d(\widehat{x}) \widetilde{\mathbf{A}}_{p, 0}(\widehat{x}) \widehat{\nabla} \widehat{u}_{1}^{(1)}+d(\widehat{x}) \widetilde{\rho}_{0}^{2}(\widehat{x}) \widehat{u}_{1}^{(1)} & =d(\widehat{x}) \widehat{f}_{0}(\widehat{x}) & & \text { in } \widehat{\Omega}, \\
\widetilde{\kappa}_{0}(x) \widehat{u}_{1}^{(1)}+\widetilde{\mathbf{A}}_{p, 0}(\widehat{x}) \widehat{\nabla} \widehat{u}_{1}^{(1)} \cdot \widehat{\boldsymbol{\nu}}_{R} & =0 & & \text { on } \widehat{\Gamma}_{R}, \\
\widehat{u}_{1}^{(1)} & =0 & & \text { on } \widehat{\Gamma}_{D}, \\
-\operatorname{div} d(\widehat{x}) \widetilde{\mathbf{A}}_{p, 2}(\widehat{x}) \widehat{\nabla} \widehat{u}_{2}^{(1)}+d(\widehat{x}) \widetilde{\rho}_{2}^{2}(\widehat{x}) \widehat{u}_{2}^{(1)} & =0 & & \\
\widetilde{\kappa}_{2}(x) \widehat{u}_{2}^{(1)}+\widetilde{\mathbf{A}}_{p, 2}(\widehat{x}) \widehat{\nabla} \widehat{u}_{2}^{(1)} \cdot \widehat{\boldsymbol{\nu}}_{R} & =0 & & \text { in } \widehat{\Omega}, \\
\widehat{u}_{2}^{(1)} & =0 & & \text { on } \widehat{\Gamma}_{R}, \\
& \text { on } \widehat{\Gamma}_{D} .
\end{array}
$$

It is obvious that in this case $\widehat{u}_{2}^{(1)}=0$, and we obtain the same system, as for the zero-order model ( $c f .(3.7))$. If we wish to improve the zero-order solution, another representation should be used, for example, one from the space

$$
\begin{aligned}
& V_{0}^{(2)}:=\left\{v \in V_{0} \mid \exists \widehat{v}_{1}, \widehat{v}_{2} \in H^{1}(\widehat{\Omega}) \text { such that } \widehat{v}_{1}=\widehat{v}_{2}=0 \text { on } \widehat{\Gamma}_{D}\right. \text { and } \\
& \left.\qquad v(x)=\widehat{v}_{1}(\widehat{x})+x_{3}^{2} \widehat{v}_{2}(\widehat{x}) \text { for a.e. } x=\left(\widehat{x}, x_{3}\right) \in \Omega\right\} .
\end{aligned}
$$

Then, the corresponding system has the form

$$
\begin{aligned}
-\operatorname{div} d(\widehat{x})\left(\widetilde{\mathbf{A}}_{p, 0}(\widehat{x}) \widehat{\nabla} \widehat{u}_{1}^{(2)}+\widetilde{\mathbf{A}}_{p, 2}(\widehat{x}) \widehat{\nabla} \widehat{u}_{2}^{(2)}\right)+d(\widehat{x})\left(\widetilde{\rho}_{0}^{2}(\widehat{x}) \widehat{u}_{1}^{(1)}+\widetilde{\rho}_{2}^{2}(\widehat{x}) \widehat{u}_{2}^{(2)}\right)=d(\widehat{x}) \widehat{f}_{0}(\widehat{x}) & \text { in } \widehat{\Omega}, \\
-\operatorname{div} d(\widehat{x})\left(\widetilde{\mathbf{A}}_{p, 2}(\widehat{x}) \widehat{\nabla} \widehat{u}_{1}^{(2)}+\widetilde{\mathbf{A}}_{p, 4}(\widehat{x}) \widehat{\nabla} \widehat{u}_{2}^{(2)}\right)+d(\widehat{x})\left(\widetilde{\rho}_{2}^{2}(\widehat{x}) \widehat{u}_{1}^{(2)}+\widetilde{\rho}_{4}^{2}(\widehat{x}) \widehat{u}_{2}^{(2)}\right)=d(\widehat{x}) \widehat{f}_{2}(\widehat{x}) & \text { in } \widehat{\Omega}, \\
\widetilde{\kappa}_{0}(x) \widehat{u}_{1}^{(2)}+\widetilde{\kappa}_{2}(x) \widehat{u}_{2}^{(2)}+\left(\widetilde{\mathbf{A}}_{p, 0}(\widehat{x}) \widehat{\nabla} \widehat{u}_{1}^{(2)}+\widetilde{\mathbf{A}}_{p, 2}(\widehat{x}) \widehat{\nabla} \widehat{u}_{2}^{(2)}\right) \cdot \widehat{\boldsymbol{\nu} R}=0 & \text { on } \widehat{\Gamma}_{R}, \\
\widetilde{\kappa}_{2}(x) \widehat{u}_{1}^{(2)}+\widetilde{\kappa}_{4}(x) \widehat{u}_{2}^{(2)}+\left(\widetilde{\mathbf{A}}_{p, 2}(\widehat{x}) \widehat{\nabla} \widehat{u}_{1}^{(2)}+\widetilde{\mathbf{A}}_{p, 4}(\widehat{x}) \widehat{\nabla} \widehat{u}_{2}^{(2)}\right) \cdot \widehat{\boldsymbol{\nu} R}=0 & \text { on } \widehat{\Gamma}_{R}, \\
\widehat{u}_{1}^{(2)}=\widehat{u}_{2}^{(2)}=0 & \text { on } \widehat{\Gamma}_{D} .
\end{aligned}
$$




\section{Estimation of the modeling error}

\subsection{Estimation of the approximation error}

Our analysis of modeling errors is based upon the so-called functional-type a posteriori error estimates (see [9] - [15] and the references cited therein). More precisely, we use such type estimate derived for the reaction-diffusion equation with mixed Dirichlet-Robin boundary conditions (see [11], estimates (4.2.21) and (4.2.22)).

Estimates are derived for the combined error norm

$$
\|u-v\|:=\left(\|\nabla(u-v)\|_{A}^{2}+\int_{\Omega} \rho^{2}(x)(u-v)^{2} d x+\left\|\kappa^{1 / 2}(u-v)\right\|_{L_{2}\left(\Gamma_{R}\right)}^{2}\right)^{1 / 2}
$$

where

$$
\|\nabla(u-v)\|_{A}^{2}:=\int_{\Omega} \mathbf{A}(x) \nabla(u-v) \cdot \nabla(u-v) d x .
$$

For convenience of the reader we recall the method, by which these estimates has been derived.

From (2.6), we obtain

$$
\begin{gathered}
\int_{\Omega}\left(\mathbf{A}(x) \nabla(u-v) \cdot \nabla w+\rho^{2}(x)(u-v) w\right) d x+\int_{\Gamma_{R}} \kappa(x)(u-v) w d s \\
=\int_{\Omega} f w d x+\int_{\Gamma_{\ominus}} F_{\ominus} w d s+\int_{\Gamma_{\oplus}} F_{\oplus} w d s \\
-\int_{\Omega}\left(\mathbf{A}(x) \nabla v \cdot \nabla w+\rho^{2}(x) v w\right) d x-\int_{\Gamma_{R}} \kappa(x) v w d s \quad \text { for all } w \in V_{0} .
\end{gathered}
$$

Let

$$
\mathbf{y} \in H^{+}(\Omega, \operatorname{Div}):=\left\{\mathbf{y} \in L_{2}\left(\Omega, \mathbb{R}^{3}\right) \mid \operatorname{Div} \mathbf{y} \in L_{2}(\Omega), \mathbf{y} \cdot \nu_{\ominus, \oplus, R} \in L_{2}\left(\Gamma_{\ominus, \oplus, R}\right)\right\} .
$$

Then

$$
\int_{\Omega} \operatorname{Div}(\mathbf{y} w) d x=\int_{\Gamma_{\ominus}}\left(\mathbf{y} \cdot \boldsymbol{\nu}_{\ominus}\right) w d s+\int_{\Gamma_{\oplus}}\left(\mathbf{y} \cdot \boldsymbol{\nu}_{\oplus}\right) w d s+\int_{\Gamma_{R}}\left(\mathbf{y} \cdot \boldsymbol{\nu}_{R}\right) w d s .
$$

On the other hand,

$$
\int_{\Omega} \operatorname{Div}(\mathbf{y} w) d x=\int_{\Omega}(w \operatorname{Div} \mathbf{y}+\mathbf{y} \cdot \nabla w) d x
$$


Set $w=u-v$. By (4.1) - (4.3), we obtain

$$
\begin{aligned}
\|u-v\|^{2}= & \int_{\Omega}\left(f-\rho^{2} v+\operatorname{Div} \mathbf{y}\right)(u-v) d x+\int_{\Gamma_{\ominus}}\left(F_{\ominus}-\mathbf{y} \cdot \boldsymbol{\nu}_{\ominus}\right)(u-v) d s \\
& +\int_{\Gamma_{\oplus}}\left(F_{\oplus}-\mathbf{y} \cdot \boldsymbol{\nu}_{R}\right)(u-v) d s \\
& +\int_{\Omega}(\mathbf{y}-\mathbf{A}(x) \nabla v) \cdot \nabla(u-v) d x-\int_{\Gamma_{R}}\left(\kappa(x) v+\mathbf{y} \cdot \boldsymbol{\nu}_{R}\right)(u-v) d s .
\end{aligned}
$$

We note that

$$
\int_{\Omega}(\mathbf{y}-\mathbf{A}(x) \nabla v) \cdot \nabla(u-v) d x \leq\|\nabla(u-v)\|_{A} M_{1}(v, \mathbf{y}),
$$

where

$$
M_{1}(v, \mathbf{y}):=\left(\int_{\Omega}\left(\nabla v-\mathbf{A}^{-1} \mathbf{y}\right) \cdot(\mathbf{A} \nabla v-\mathbf{y}) d x\right)^{1 / 2}
$$

represents the part of error associated with violation of the relation $p=\mathbf{A} \nabla u$ (which holds for the exact solution and the corresponding flux $p$ ).

Let

$$
M_{2}(v, \mathbf{y}):=\left\|\operatorname{Div} \mathbf{y}-\rho^{2} v+f\right\|_{L_{2}(\Omega)},
$$

and

$$
\begin{aligned}
& M_{3 R}(v, \mathbf{y}):=\left\|\kappa v+\mathbf{y} \cdot \boldsymbol{\nu}_{R}\right\|_{L_{2}\left(\Gamma_{R}\right)}, \\
& M_{3 \ominus}(v, \mathbf{y}):=\left\|F_{\ominus}-\mathbf{y} \cdot \boldsymbol{\nu}_{\ominus}\right\|_{L_{2}\left(\Gamma_{\ominus}\right)}, \\
& M_{3 \oplus}(v, \mathbf{y}):=\left\|F_{\oplus}-\mathbf{y} \cdot \boldsymbol{\nu}_{\oplus}\right\|_{L_{2}\left(\Gamma_{\oplus}\right)} .
\end{aligned}
$$

These functionals correspond to the parts of the error generated by inconveniences in the differential equation and boundary conditions, respectively. It is easy to see that

$$
\int_{\Omega}\left(f-\rho^{2} v+\operatorname{Divy}\right)(u-v) d x \leq C_{F, \Omega} c_{1}^{-1} M_{2}(v, \mathbf{y})\|\nabla(u-v)\|_{A},
$$

where

$$
C_{F, \Omega}^{-2}:=\inf _{w \in V_{0} \backslash\{0\}} \frac{\|\nabla w\|_{A}^{2}}{\|w\|_{L_{2}(\Omega)}^{2}} .
$$

Another way to estimate this integral from above arises if $\rho$ is a strictly positive function. Then,

$$
\int_{\Omega}\left(f-\rho^{2} v+\operatorname{Divy}\right)(u-v) d x \leq \widetilde{M}_{2}(v, \mathbf{y})\|\rho(u-v)\|_{L_{2}(\Omega)},
$$


where

$$
\widetilde{M}_{2}(v, \mathbf{y}):=\left\|\rho^{-1}\left(\operatorname{Div} \mathbf{y}-\rho^{2} v+f\right)\right\|_{L_{2}(\Omega)}
$$

Analogously,

$$
\begin{gathered}
\int_{\Gamma_{\ominus}}\left(F_{\ominus}-\mathbf{y} \cdot \boldsymbol{\nu}_{\ominus}\right)(u-v) d s \leq C_{\Gamma \ominus} c_{1}^{-1} M_{3 \ominus}(v, \mathbf{y})\|\nabla(u-v)\|_{A}, \\
\int_{\Gamma_{\oplus}}\left(F_{\oplus}-\mathbf{y} \cdot \boldsymbol{\nu}_{\oplus}\right)(u-v) d s \leq C_{\Gamma \oplus} c_{1}^{-1} M_{3 \oplus}(v, \mathbf{y})\|\nabla(u-v)\|_{A},
\end{gathered}
$$

where the constants $C_{\Gamma \ominus}$ and $C_{\Gamma \oplus}$ come from the trace inequalities

$$
\|w\|_{L_{2}\left(\Gamma_{\ominus}, \Gamma_{\oplus}\right)}^{2} \leq C_{\Gamma \ominus, \oplus}\|\nabla w\|
$$

Finally, we note that the boundary integral on $\Gamma_{R}$ can be estimated by two ways. The first one is also based on the corresponding trace inequality

$$
\int_{\Gamma_{R}}\left(\kappa(x) v+\mathbf{y} \cdot \boldsymbol{\nu}_{R}\right)(u-v) d s \leq C_{\Gamma_{R}} c_{1}^{-1} M_{3 R}(v, \mathbf{y})\|\nabla(u-v)\|_{A} .
$$

If $\kappa$ is strictly positive, we apply another estimate

$$
\int_{\Gamma_{R}}\left(\kappa(x) v+\mathbf{y} \cdot \boldsymbol{\nu}_{R}\right)(u-v) d s \leq \widetilde{M}_{3 R}(v, \mathbf{y})\left\|\kappa^{1 / 2}(u-v)\right\|_{L_{2}\left(\Gamma_{R}\right)},
$$

where

$$
\widetilde{M}_{3 R}(v, \mathbf{y}):=\left\|\kappa^{-1 / 2}\left(\kappa v+\mathbf{y} \cdot \boldsymbol{\nu}_{R}\right)\right\|_{L_{2}\left(\Gamma_{R}\right)} .
$$

By (4.4) - (4.7) and (4.10) - (4.12), we arrive at the estimate

$$
\begin{aligned}
\|u-v\| \leq M_{1}(v, \mathbf{y}) & +C_{F, \Omega} c_{1}^{-1} M_{2}(v, \mathbf{y}) \\
& +c_{1}^{-1}\left(C_{\Gamma_{\ominus}} M_{3 \ominus}(v, \mathbf{y})+C_{\Gamma_{\oplus}} M_{3 \oplus}(v, \mathbf{y})+C_{\Gamma_{R}} M_{3 R}(v, \mathbf{y})\right) .
\end{aligned}
$$

By (4.4) - (4.6), (4.8) - (4.12), we obtain for a strictly positive function $\rho$ another estimate

$$
\begin{aligned}
\|u-v\| \leq \sqrt{M_{1}^{2}(v, \mathbf{y})+\widetilde{M}_{2}^{2}(v, \mathbf{y})} & \\
& \quad+c_{1}^{-1}\left(C_{\Gamma_{\ominus}} M_{3 \ominus}(v, \mathbf{y})+C_{\Gamma_{\oplus}} M_{3 \oplus}(v, \mathbf{y})+C_{\Gamma_{R}} M_{3 R}(v, \mathbf{y})\right) .
\end{aligned}
$$

If $(4.13)$ - (4.14) is used instead of (4.12), then

$$
\begin{aligned}
& \|u-v\| \leq \sqrt{M_{1}^{2}(v, \mathbf{y})+\widetilde{M}_{2}^{2}(v, \mathbf{y})+\widetilde{M}_{3 R}^{2}(v, \mathbf{y})} \\
& \left.+c_{1}^{-1}\left(C_{\Gamma_{\ominus}} M_{3 \ominus}(v, \mathbf{y})+C_{\Gamma_{\oplus}} M_{3 \oplus}(v, \mathbf{y})\right)\right) .
\end{aligned}
$$

We summarize the results in the following 
Theorem 4.1 (i) For any $v \in V_{0}$ and any $\mathbf{y} \in H^{+}(\Omega$, Div) the estimate (4.15) provides an upper bound of the distance to the exact solution $u$ measured in terms of the natural energy norm.

(ii) If $\rho(x)>0$, then (4.16) suggests another upper bound, which does not involve the constant $C_{F, \Omega}$.

(iii) If both functions $\rho$ and $\kappa$ are strictly positive, then (4.17) suggests an upper bound that does not contain $C_{F, \Omega}$ and $C_{\Gamma, R}$.

(iv) If, in addition,

$$
\mathbf{y} \in H_{\ominus, \oplus}^{+}(\Omega, \operatorname{Div}):=\left\{\mathbf{y} \in L_{2}\left(\Omega, \mathbb{R}^{3}\right) \mid \operatorname{Div} \mathbf{y} \in L_{2}(\Omega), \mathbf{y} \cdot \nu_{\ominus, \oplus}=F_{\ominus, \oplus}\right\},
$$

then the distance to $u$ is subject to the estimate

$$
\|u-v\|^{2} \leq M_{1}^{2}(v, \mathbf{y})+\widetilde{M}_{2}^{2}(v, \mathbf{y})+\widetilde{M}_{3 R}^{2}(v, \mathbf{y}),
$$

which contains no constants.

\subsection{Error of zero-order model}

We apply the results obtained in the previous section in order to measure modeling errors associated with zero and first order models. In what follows, we use the following notation

$$
\begin{aligned}
& \mathbf{B}:=\mathbf{A}^{-1} \quad\left(\mathbf{B}(x)=\left(b_{i j}(x)\right)_{i, j=1,3}, \quad \mathbf{B}=\mathbf{B}^{T}\right), \\
& \mathbf{B}_{p, 0}:=\left(b_{i j}\right)_{i, j=1,2}, \quad \widetilde{\mathbf{B}}_{p, 0}(\widehat{x}):=\frac{1}{d(\widehat{x})} \int_{d_{\ominus}(\widehat{x})}^{d_{\oplus}(\widehat{x})} \mathbf{B}_{p, 0}(x) d x_{3}, \\
& \mathbf{a}_{3}:=\left\{a_{31}, a_{32}, a_{33}\right\}^{T}, \quad \mathbf{b}_{3}:=\left\{b_{31}, b_{32}, b_{33}\right\}^{T} .
\end{aligned}
$$

Theorem 4.2 (i) The error of the zero-order reduced model is subject to the following estimate:

$$
\left\|u-\widehat{u}^{(0)}\right\| \leq M_{1}+M_{2}+M_{3}
$$

where

$$
\begin{aligned}
M_{1}:=\left(\int_{\widehat{\Omega}} d(\widehat{x})\left(\widetilde{\mathbf{B}}_{p, 0} \widetilde{\mathbf{A}}_{p, 0}-\mathbf{I}\right) \widehat{\nabla} \widehat{u}^{(0)} \cdot \widetilde{\mathbf{A}}_{p, 0} \widehat{\nabla} \widehat{u}^{(0)} d \widehat{x}\right. \\
\left.\quad+\int_{\Omega}\left(b_{33} \psi(x)^{2}+2\left(\mathbf{b}_{3} \cdot\left(\widetilde{\mathbf{A}}_{p, 0} \widehat{\nabla} \widehat{u}^{(0)}, 0\right)\right) \psi(x)\right) d x\right)^{1 / 2},
\end{aligned}
$$




$$
M_{2}:=C_{F, \Omega} c_{1}^{-1}\left\|\left(f-\widehat{f}_{0}\right)-\left(\rho^{2}-\widetilde{\rho}_{0}^{2}\right) \widehat{u}^{(0)}-\frac{\nabla d(\widehat{x})}{d(\widehat{x})} \cdot \widetilde{\mathbf{A}}_{p, 0} \widehat{\nabla} \widehat{u}^{(0)}+\frac{\partial \psi}{\partial x_{3}}\right\|_{L_{2}(\Omega)}(4
$$

and

$$
\begin{aligned}
M_{3}:=c_{1}^{-1} & \left(C_{\Gamma, R}\left\|\kappa \widehat{u}^{(0)}+\left(\widetilde{\mathbf{A}}_{p, 0} \widehat{\nabla} \widehat{u}^{(0)}, 0\right) \cdot \boldsymbol{\nu}_{R}\right\|_{L_{2}\left(\Gamma_{R}\right)}\right. \\
& +C_{\Gamma, \ominus}\left\|F_{\ominus}-\left(\widetilde{\mathbf{A}}_{p, 0} \widehat{\nabla} \widehat{u}^{(0)}, 0\right) \cdot \boldsymbol{\nu}_{\ominus}-\psi \nu_{\ominus 3}\right\|_{L_{2}\left(\Gamma_{\ominus}\right)} \\
& \left.+C_{\Gamma, \oplus}\left\|F_{\oplus}-\left(\widetilde{\mathbf{A}}_{p, 0} \widehat{\nabla} \widehat{u}^{(0)}, 0\right) \cdot \boldsymbol{\nu}_{\oplus}-\psi \nu_{\oplus 3}\right\|_{L_{2}\left(\Gamma_{\oplus}\right)}\right)
\end{aligned}
$$

with an auxiliary function $\psi$ satisfying the conditions

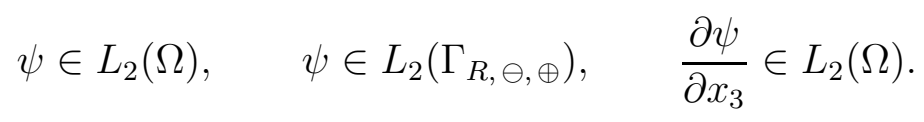

(ii) Assume $\rho$ strictly positive. Then (4.21) can be modified to the estimate

$$
\left\|u-\widehat{u}^{(0)}\right\| \leq \sqrt{M_{1}^{2}+\widetilde{M_{2}^{2}}}+M_{3}
$$

where $M_{1}$ and $M_{3}$ are from (4.22) and (4.24), respectively, and the functional $\widetilde{M}_{2}^{2}$ is defined by

$$
\widetilde{M}_{2}^{2}:=\left\|\rho^{-1}\left(\left(f-\widehat{f}_{0}\right)-\left(\rho^{2}-\widetilde{\rho}_{0}^{2}\right) \widehat{u}^{(0)}-\frac{\nabla d(\widehat{x})}{d(\widehat{x})} \cdot \widetilde{\mathbf{A}}{ }_{p, 0} \widehat{\nabla} \widehat{u}^{(0)}+\frac{\partial \psi}{\partial x_{3}}\right)\right\|_{L_{2}(\Omega)}^{2} .
$$

(iii) If the function $\kappa$ is also strictly positive, then we obtain an upper bound

$$
\left\|u-\widehat{u}^{(0)}\right\| \leq \sqrt{M_{1}^{2}+\widetilde{M_{2}^{2}}+\widetilde{M}_{3 R}^{2}}+M_{3 \ominus \oplus},
$$

where $M_{1}$ and $\widetilde{M}_{2}^{2}$ are from (4.22) and (4.27), respectively, and

$$
\begin{aligned}
\widetilde{M}_{3 R}:= & \left\|\kappa^{-1 / 2}\left(\kappa \widehat{u}^{(0)}+\left(\widetilde{\mathbf{A}}_{p, 0} \widehat{\nabla} \widehat{u}^{(0)}, 0\right) \cdot \boldsymbol{\nu}_{R}\right)\right\|_{L_{2}\left(\Gamma_{R}\right)}, \\
M_{3 \oplus \ominus}:=c_{1}^{-1} & \left(C_{\Gamma, \ominus}\left\|F_{\ominus}-\left(\widetilde{\mathbf{A}}_{p, 0} \widehat{\nabla} \widehat{u}^{(0)}, 0\right) \cdot \boldsymbol{\nu}_{\ominus}-\psi \nu_{\ominus 3}\right\|_{L_{2}\left(\Gamma_{\ominus}\right)}\right. \\
& \left.+C_{\Gamma, \oplus}\left\|F_{\oplus}-\left(\widetilde{\mathbf{A}}_{p, 0} \widehat{\nabla} \widehat{u}^{(0)}, 0\right) \cdot \boldsymbol{\nu}_{\oplus}-\psi \nu_{\oplus 3}\right\|_{L_{2}\left(\Gamma_{\oplus}\right)}\right) .
\end{aligned}
$$

Proof: $(i)$ We use (4.15) with $v=\widehat{u}^{(0)} \in V_{0}$. Certainly, the best possible option would be to take $\mathbf{y}$ as the exact flux $\mathbf{A} \nabla u$, but we have to restrict ourselves to choosing a quantity that we can obtain with the help of dimensionally reduced model, i.e., not containing the unknown exact solution $u$. For this reason, we approximate the flux by

$$
\mathbf{y}=\left(\widetilde{\mathbf{A}}_{p, 0} \widehat{\nabla} \widehat{u}^{(0)}, 0\right)+\boldsymbol{\tau} \quad \text { with } \quad \boldsymbol{\tau}=\{0,0, \psi(x)\}^{T},
$$

where the auxiliary function $\psi$ satisfies the conditions (4.25). Concrete forms of the function $\psi$ will to be discussed later (see Remark 4.3). One can easily verify that the 
vector valued function $\mathbf{y}$ defined by (4.31) belongs to $H^{+}(\Omega$, Div).

Using the notation (4.18)-(4.20) we represent the functional $M_{1}$ in the form

$$
\begin{aligned}
M_{1} & =\left(\int_{\Omega}\left(\left(\widehat{\nabla} \widehat{u}^{(0)}, 0\right)-\mathbf{B y}\right) \cdot\left(\mathbf{A}\left(\widehat{\nabla} \widehat{u}^{(0)}, 0\right)-\mathbf{y}\right) d x\right)^{1 / 2} \\
& =\left(\int_{\Omega} \mathbf{A}\left(\widehat{\nabla} \widehat{u}^{(0)}, 0\right) \cdot\left(\widehat{\nabla} \widehat{u}^{(0)}, 0\right) d x-2 \int_{\Omega} \mathbf{y} \cdot\left(\widehat{\nabla} \widehat{u}^{(0)}, 0\right) d x+\int_{\Omega} \mathbf{B y} \cdot \mathbf{y} d x\right)^{1 / 2} .
\end{aligned}
$$

It is clear that

$$
\int_{\Omega} \mathbf{A}\left(\widehat{\nabla} \widehat{u}^{(0)}, 0\right) \cdot\left(\widehat{\nabla} \widehat{u}^{(0)}, 0\right) d x=\int_{\widehat{\Omega}} d(\widehat{x}) \widetilde{\mathbf{A}}_{p, 0}(\widehat{x}) \widehat{\nabla} \widehat{u}^{(0)} \cdot \widehat{\nabla} \widehat{u}^{(0)} d \widehat{x}
$$

and

$$
\begin{aligned}
\int_{\Omega} \mathbf{y} \cdot\left(\widehat{\nabla} \widehat{u}^{(0)}, 0\right) d x & =\int_{\Omega}\left(\left(\widetilde{\mathbf{A}}_{p, 0} \widehat{\nabla} \widehat{u}^{(0)}, 0\right)+\tau\right) \cdot\left(\widehat{\nabla} \widehat{u}^{(0)}, 0\right) d x \\
& =\int_{\widehat{\Omega}} d(\widehat{x}) \widetilde{\mathbf{A}_{p, 0}}(\widehat{x}) \widehat{\nabla} \widehat{u}^{(0)} \cdot \widehat{\nabla} \widehat{u}^{(0)} d \widehat{x}
\end{aligned}
$$

Since

$$
\begin{aligned}
\mathbf{B y} \cdot \mathbf{y}= & \left(\mathbf{B}\left(\widetilde{\mathbf{A}}_{p, 0} \widehat{\nabla} \widehat{u}^{(0)}, 0\right)+\mathbf{B} \tau\right) \cdot\left(\left(\widetilde{\mathbf{A}}_{p, 0} \widehat{\nabla} \widehat{u}^{(0)}, 0\right)+\tau\right) \\
= & \mathbf{B}\left(\widetilde{\mathbf{A}}_{p, 0} \widehat{\nabla} \widehat{u}^{(0)}, 0\right) \cdot\left(\widetilde{\mathbf{A}}_{p, 0} \widehat{\nabla} \widehat{u}^{(0)}, 0\right)+\mathbf{B}\left(\widetilde{\mathbf{A}}_{p, 0} \widehat{\nabla} \widehat{u}^{(0)}, 0\right) \cdot \tau \\
& +\mathbf{B} \tau \cdot\left(\widetilde{\mathbf{A}}_{p, 0} \widehat{\nabla} \widehat{u}^{(0)}, 0\right)+\mathbf{B} \tau \cdot \tau \\
= & \mathbf{B}_{p, 0} \widetilde{\mathbf{A}}_{p, 0} \widehat{\nabla} \widehat{u}^{(0)} \cdot \widetilde{\mathbf{A}}_{p, 0} \widehat{\nabla} \widehat{u}^{(0)}+2\left(\mathbf{b}_{3} \cdot\left(\widetilde{\mathbf{A}}_{p, 0} \widehat{\nabla} \widehat{u}^{(0)}, 0\right)\right) \psi+b_{33} \psi^{2},
\end{aligned}
$$

we see that

$$
\begin{aligned}
\int_{\Omega} \mathbf{B y} \cdot \mathbf{y} d x=\int_{\widehat{\Omega}} d(\widehat{x}) \widetilde{\mathbf{B}}_{p, 0} \widetilde{\mathbf{A}}_{p, 0} \widehat{\nabla} \widehat{u}^{(0)} \cdot \widetilde{\mathbf{A}}_{p, 0} \widehat{\nabla} \widehat{u}^{(0)} d \widehat{x} \\
\quad+\int_{\Omega}\left(b_{33} \psi^{2}+2\left(\mathbf{b}_{3} \cdot\left(\widetilde{\mathbf{A}}_{p, 0} \widehat{\nabla} \widehat{u}^{(0)}, 0\right)\right) \psi\right) d x .
\end{aligned}
$$

Now (4.33), (4.34) and (4.35) imply

$$
\begin{aligned}
M_{1}=\left(\int_{\widehat{\Omega}} d(\widehat{x})\left(\widetilde{\mathbf{B}}_{p, 0} \widetilde{\mathbf{A}}_{p, 0}-\mathbf{I}\right) \widehat{\nabla} \widehat{u}^{(0)} \cdot \widetilde{\mathbf{A}}_{p, 0} \widehat{\nabla} \widehat{u}^{(0)} d \widehat{x}\right. \\
\left.\quad+\int_{\Omega}\left(b_{33} \psi^{2}+2\left(\mathbf{b}_{3} \cdot\left(\widetilde{\mathbf{A}}_{p, 0} \widehat{\nabla} \widehat{u}^{(0)}, 0\right)\right) \psi\right) d x\right)^{1 / 2},
\end{aligned}
$$


where $\mathbf{I}$ is the identity $2 \times 2$-matrix.

Consider the functional $M_{2}$. Since $\mathbf{y}$ is chosen as in (4.31), we find that

$$
\operatorname{Div} \mathbf{y}=\operatorname{div} \widetilde{\mathbf{A}}_{p, 0} \widehat{\nabla} \widehat{u}^{(0)}+\operatorname{Div} \tau=\operatorname{div} \widetilde{\mathbf{A}}_{p, 0} \widehat{\nabla} \widehat{u}^{(0)}+\frac{\partial \psi}{\partial x_{3}}
$$

From the product rule for the divergence, it follows that

$$
\operatorname{div} d(\widehat{x}) \widetilde{\mathbf{A}}_{p, 0} \widehat{\nabla} \widehat{u}^{(0)}=\nabla d(\widehat{x}) \cdot \widetilde{\mathbf{A}}_{p, 0} \widehat{\nabla} \widehat{u}^{(0)}+d(\widehat{x}) \operatorname{div} \widetilde{\mathbf{A}}_{p, 0} \widehat{\nabla} \widehat{u}^{(0)} .
$$

Now we conclude with (3.7)

$$
\operatorname{div} \widetilde{\mathbf{A}}_{p, 0} \widehat{\nabla} \widehat{u}^{(0)}=-\frac{\nabla d(\widehat{x})}{d(\widehat{x})} \cdot \widetilde{\mathbf{A}}_{p, 0} \widehat{\nabla} \widehat{u}^{(0)}+\left(\widehat{\rho}_{0}^{2} \widehat{u}^{(0)}-\widehat{f}_{0}\right) .
$$

Thus, the term $M_{2}$ reads with (3.3) - (3.5)

$$
M_{2}=C_{F, \Omega} c_{1}^{-1}\left\|\left(f-\widehat{f}_{0}\right)-\left(\rho^{2}-\widetilde{\rho}_{0}^{2}\right) \widehat{u}^{(0)}-\frac{\nabla d(\widehat{x})}{d(\widehat{x})} \cdot \widetilde{\mathbf{A}}_{p, 0} \widehat{\nabla} \widehat{u}^{(0)}+\frac{\partial \psi}{\partial x_{3}}\right\|_{L_{2}(\Omega)} .
$$

Finally, consider the functional $M_{3}$. Since the third component of the normal vectors $\boldsymbol{\nu}_{R}$ is zero, we obtain

$$
\begin{aligned}
M_{3}=c_{1}^{-1}( & C_{\Gamma, R}\left\|\kappa \widehat{u}^{(0)}+\left(\widetilde{\mathbf{A}}_{p, 0} \widehat{\nabla} \widehat{u}^{(0)}, 0\right) \cdot \boldsymbol{\nu}_{R}\right\|_{L_{2}\left(\Gamma_{R}\right)} \\
& +C_{\Gamma, \ominus}\left\|F_{\ominus}-\left(\widetilde{\mathbf{A}}_{p, 0} \widehat{\nabla} \widehat{u}^{(0)}, 0\right) \cdot \boldsymbol{\nu}_{\ominus}-\psi \nu_{\ominus 3}\right\|_{L_{2}\left(\Gamma_{\ominus}\right)} \\
& \left.+C_{\Gamma, \oplus}\left\|F_{\oplus}-\left(\widetilde{\mathbf{A}}_{p, 0} \widehat{\nabla} \widehat{u}^{(0)}, 0\right) \cdot \boldsymbol{\nu}_{\oplus}-\psi \nu_{\oplus 3}\right\|_{L_{2}\left(\Gamma_{\oplus}\right)}\right)
\end{aligned}
$$

where

$$
\nu_{\ominus 3}=\frac{-1}{\sqrt{1+\left|\nabla d_{\ominus}\right|^{2}}}, \quad \nu_{\oplus 3}=\frac{1}{\sqrt{1+\left|\nabla d_{\oplus}\right|^{2}}}
$$

are the third components of the normal vectors $\boldsymbol{\nu}_{\ominus}$ and $\boldsymbol{\nu}_{\oplus}$.

(ii) The estimate (4.26) with $\widetilde{M}_{2}^{2}$ from (4.27) follows from Theorem 4.1 (ii) and (4.37) - (4.39).

(iii) The statement follows from Theorem 4.1 (iii) and (4.41).

Remark 4.1 Theorem 4.2 (i) provides a general a posteriori estimate for the dimension reduction error in the case of the zero-order model. Three terms in the right hand side have a clear meaning: $M_{1}$ evaluates the error generated by the fact that $\widehat{\nabla} \widehat{u}^{(0)}$ and the reconstructed flux $\mathbf{y}$ (cf. (4.31)) does not exactly satisfy the three-dimensional relation, $M_{2}$ represents the error in the equilibrium equation, and $M_{3}$ the error in the boundary condition. We note that for the stationary diffusion problem such an estimate has been earlier derived and tested in [15].

Remark 4.2 It is worth to notice that the error estimate (4.26) does not involve the constant $C_{F, \Omega}$ in the case of $\rho(x)>0$. If additionally $\kappa(x)>0$, the estimate contains neither $C_{F, \Omega}$ nor $C_{\Gamma, R}$ (cf. (4.29) - (4.30)). 
Remark 4.3 It is necessary to discuss an appropriate form of the auxiliary function $\psi$. We can follow the recommendation from [15] and choose such $\psi$ that the last two terms of $M_{3}$ in (4.24) (i.e., the residual on the Neumann boundary conditions) are identically zero. We note that

$$
\begin{aligned}
\left\|F_{\ominus}-\left(\widetilde{\mathbf{A}}_{p, 0} \nabla \widehat{u}^{(0)}, 0\right) \cdot \boldsymbol{\nu}_{\ominus}-\psi \nu_{\ominus 3}\right\|_{\Gamma_{\ominus}}^{2}= & \int_{\widehat{\Omega}}\left(\widehat{F}_{\ominus}(\widehat{x})-\left(\widetilde{\mathbf{A}}_{p, 0} \widehat{\nabla} \widehat{u}^{(0)}, 0\right) \cdot \boldsymbol{\nu}_{\ominus}\right. \\
& \left.-\psi\left(\widehat{x}, d_{\ominus}(\widehat{x})\right) \nu_{\ominus 3}\right)^{2} \sqrt{1+\left|\nabla d_{\ominus}\right|^{2}} d \widehat{x}
\end{aligned}
$$

(analogously for the norm in $L_{2}\left(\Gamma_{\oplus}\right)$ ) and set

$$
\psi(x)=\psi_{1}(x):=\widehat{\alpha}(\widehat{x}) x_{3}+\widehat{\beta}(\widehat{x}),
$$

where the functions $\widehat{\alpha}$ and $\widehat{\beta}\left(\widehat{\alpha}, \widehat{\beta} \in L_{2}(\widehat{\Omega})\right)$ are uniquely defined by the condition

$$
\psi \nu_{\oplus, \ominus 3}=\widehat{F}_{\oplus, \ominus}-\left(\widetilde{\mathbf{A}}_{p, 0} \widehat{\nabla} \widehat{u}^{(0)}, 0\right) \cdot \boldsymbol{\nu}_{\oplus, \ominus} \text { at } x_{3}=d_{\oplus, \ominus}
$$

(cf. [15]). The corresponding $\widehat{\alpha}$ and $\widehat{\beta}$ have the form

$$
\widehat{\alpha}=\frac{1}{d}\left(\frac{\widehat{G}_{\oplus}}{\nu_{\oplus 3}}-\frac{\widehat{G}_{\ominus}}{\nu_{\ominus 3}}\right) \quad \text { and } \quad \widehat{\beta}=\frac{1}{d}\left(\frac{\widehat{G}_{\ominus}}{\nu_{\ominus 3}} d_{\oplus}-\frac{\widehat{G}_{\oplus}}{\nu_{\oplus 3}} d_{\ominus}\right)
$$

with

$$
\widehat{G}_{\oplus, \ominus}:=\widehat{F}_{\oplus, \ominus}-\left(\widetilde{\mathbf{A}}_{p, 0} \widehat{\nabla} \widehat{u}^{(0)}, 0\right) \cdot \boldsymbol{\nu}_{\oplus, \ominus} .
$$

It is easy to see that $\widehat{\alpha}, \widehat{\beta} \in L_{2}(\widehat{\Omega})$. Hence, $\psi$ from (4.42) and its derivative in $x_{3}$ direction belongs to $L_{2}(\Omega)$.

With (4.42)- (4.44) we obtain for the right hand side terms of (4.21) the following representation:

$$
\begin{aligned}
& M_{1}=\left(\int_{\widehat{\Omega}} d(\widehat{x})\left(\widetilde{\mathbf{B}}_{p, 0} \widetilde{\mathbf{A}}_{p, 0}-\mathbf{I}\right) \widehat{\nabla} \widehat{u}^{(0)} \cdot \widetilde{\mathbf{A}}_{p, 0} \widehat{\nabla} \widehat{u}^{(0)} d \widehat{x}\right. \\
& \left.\quad+\int_{\Omega}\left(b_{33}\left(\widehat{\alpha}(\widehat{x}) x_{3}+\widehat{\beta}(\widehat{x})\right)^{2}+2\left(\mathbf{b}_{3} \cdot\left(\widetilde{\mathbf{A}}_{p, 0} \widehat{\nabla} \widehat{u}^{(0)}, 0\right)\right)\left(\widehat{\alpha}(\widehat{x}) x_{3}+\widehat{\beta}(\widehat{x})\right)\right) d x\right)^{1 / 2} \\
& M_{2}=C_{F, \Omega} c_{1}^{-1}\left\|\left(f-\widehat{f}_{0}\right)-\left(\rho^{2}-\widetilde{\rho}_{0}^{2}\right) \widehat{u}^{(0)}-\frac{\nabla d(\widehat{x})}{d(\widehat{x})} \cdot \widetilde{\mathbf{A}}_{p, 0} \widehat{\nabla} \widehat{u}^{(0)}+\widehat{\alpha}\right\|_{L_{2}(\Omega)}
\end{aligned}
$$

and

$$
\begin{aligned}
M_{3}=c_{1}^{-1}( & C_{\Gamma, R}\left\|\kappa \widehat{u}^{(0)}+\left(\widetilde{\mathbf{A}}_{p, 0} \widehat{\nabla} \widehat{u}^{(0)}, 0\right) \cdot \boldsymbol{\nu}_{R}\right\|_{L_{2}\left(\Gamma_{R}\right)} \\
& +C_{\Gamma, \ominus}\left\|F_{\ominus}-\left(\widetilde{\mathbf{A}}_{p, 0} \widehat{\nabla} \widehat{u}^{(0)}, 0\right) \cdot \boldsymbol{\nu}_{\ominus}-\left(\widehat{\alpha}(\widehat{x}) x_{3}+\widehat{\beta}(\widehat{x})\right) \nu_{\ominus 3}\right\|_{L_{2}\left(\Gamma_{\ominus}\right)} \\
& \left.+C_{\Gamma, \oplus}\left\|F_{\oplus}-\left(\widetilde{\mathbf{A}}_{p, 0} \widehat{\nabla} \widehat{u}^{(0)}, 0\right) \cdot \boldsymbol{\nu}_{\oplus}-\left(\widehat{\alpha}(\widehat{x}) x_{3}+\widehat{\beta}(\widehat{x})\right) \nu_{\oplus 3}\right\|_{L_{2}\left(\Gamma_{\oplus}\right)}\right) .
\end{aligned}
$$




\subsection{Error of first-order model}

Theorem 4.3 (i) The error of the first-order reduced model is subject to the following estimate:

$$
\left\|u-\widehat{u}^{(1)}\right\| \leq M_{1}+M_{2}+M_{3}
$$

with

$$
\begin{aligned}
& M_{1}:= \int_{\Omega}\left[\widehat{u}_{2}^{(1)}\left(2 \mathbf{a}_{3} \cdot\left(\widehat{\nabla} \widehat{u}_{1}^{(1)}+x_{3} \widehat{\nabla} \widehat{u}_{2}^{(1)}, 0\right)+a_{33} \widehat{u}_{2}^{(1)}-\psi\right)+b_{33} \psi^{2}\right. \\
&\left.+2\left(\mathbf{b}_{3} \cdot\left(\widetilde{\mathbf{A}}_{p, 0} \widehat{\nabla} \widehat{u}_{1}^{(1)}+\widetilde{\mathbf{A}}_{p, 1} \widehat{\nabla} \widehat{u}_{2}^{(1)}, 0\right)\right) \psi\right] d x \\
&+\int_{\widehat{\Omega}} d(\widehat{x})\left[\widetilde{\mathbf{B}}_{p, 0}\left(\left(\widetilde{\mathbf{A}}_{p, 0}-\widetilde{\mathbf{B}}_{p, 0}^{-1}\right) \widehat{\nabla} \widehat{u}_{1}^{(1)}+\widetilde{\mathbf{A}}_{p, 1} \widehat{\nabla} \widehat{u}_{2}^{(1)}\right) \cdot\left(\widetilde{\mathbf{A}}_{p, 0} \widehat{\nabla} \widehat{u}_{1}^{(1)}+\widetilde{\mathbf{A}}_{p, 1} \widehat{\nabla} \widehat{u}_{2}^{(1)}\right)\right. \\
&\left.\left.+\widehat{\nabla} \widehat{u}_{2}^{(1)} \cdot\left(\widetilde{\mathbf{A}}_{p, 1} \widehat{\nabla} \widehat{u}_{1}^{(1)}+\widetilde{\mathbf{A}}_{p, 2} \widehat{\nabla} \widehat{u}_{2}^{(1)}\right)\right] d \widehat{x}\right)^{1 / 2}, \\
& M_{2}:= C_{F, \Omega} c_{1}^{-1} \|\left(f-\widehat{f}_{0}\right)-\left(\rho^{2}-\widetilde{\rho}_{0}^{2}\right) \widehat{u}_{1}^{(1)}-\left(x_{3} \rho^{2}-\widetilde{\rho}_{1}^{2}\right) \widehat{u}_{2}^{(1)} \\
& \quad-\frac{\nabla d(\widehat{x})}{d(\widehat{x})} \cdot\left(\widetilde{\mathbf{A}}_{p, 0} \widehat{\nabla} \widehat{u}_{1}^{(1)}+\widetilde{\mathbf{A}}_{p, 1} \widehat{\nabla} \widehat{u}_{2}^{(1)}\right)+\frac{\partial \psi}{\partial x_{3}} \|_{L_{2}(\Omega)},
\end{aligned}
$$

and

$$
\begin{aligned}
M_{3} & :=c_{1}^{-1}\left(C_{\Gamma, R}\left\|\kappa\left(\widehat{u}_{1}^{(1)}+x_{3} \widehat{u}_{2}^{(1)}\right)+\left(\widetilde{\mathbf{A}}_{p, 0} \widehat{\nabla} \widehat{u}_{1}^{(1)}+\widetilde{\mathbf{A}}_{p, 1} \widehat{\nabla} \widehat{u}_{2}^{(1)}, 0\right) \cdot \boldsymbol{\nu}_{R}\right\|_{L_{2}\left(\Gamma_{R}\right)}\right. \\
& +C_{\Gamma, \ominus}\left\|F_{\ominus}-\left(\widetilde{\mathbf{A}}_{p, 0} \widehat{\nabla} \widehat{u}_{1}^{(1)}+\widetilde{\mathbf{A}}_{p, 1} \widehat{\nabla} \widehat{u}_{2}^{(1)}, 0\right) \cdot \boldsymbol{\nu}_{\ominus}-\psi \nu_{\ominus 3}\right\|_{L_{2}\left(\Gamma_{\ominus}\right)} \\
& \left.+C_{\Gamma, \oplus}\left\|F_{\oplus}-\left(\widetilde{\mathbf{A}}_{p, 0} \widehat{\nabla} \widehat{u}_{1}^{(1)}+\widetilde{\mathbf{A}}_{p, 1} \widehat{\nabla} \widehat{u}_{2}^{(1)}, 0\right) \cdot \boldsymbol{\nu}_{\oplus}-\psi \nu_{\oplus 3}\right\|_{L_{2}\left(\Gamma_{\oplus}\right)}\right),
\end{aligned}
$$

where definitions (3.4) and (4.18) - (4.20) are used, and $\psi$ is an auxiliary function satisfying the conditions

$$
\psi \in L_{2}(\Omega), \quad \psi \in L_{2}\left(\Gamma_{R, \ominus, \oplus}\right), \quad \frac{\partial \psi}{\partial x_{3}} \in L_{2}(\Omega) .
$$

(ii) Assume $\rho$ strictly positive. Then (4.48) can be modified to the estimate that does not involve the constant $C_{F, \Omega}$ :

$$
\left\|u-\widehat{u}^{(1)}\right\| \leq \sqrt{M_{1}^{2}+\widetilde{M}_{2}^{2}}+M_{3}
$$

where $M_{1}$ and $M_{3}$ are from (4.49) and (4.51), respectively, and the functional $\widetilde{M}_{2}^{2}$ is defined by

$$
\begin{aligned}
\widetilde{M}_{2}^{2}:=\| \rho^{-1}\left(\left(f-\widehat{f}_{0}\right)\right. & -\left(\rho^{2}-\widetilde{\rho}_{0}^{2}\right) \widehat{u}_{1}^{(1)}-\left(x_{3} \rho^{2}-\widetilde{\rho}_{1}^{2}\right) \widehat{u}_{2}^{(1)} \\
& \left.-\frac{\nabla d(\widehat{x})}{d(\widehat{x})} \cdot\left(\widetilde{\mathbf{A}}_{p, 0} \widehat{\nabla} \widehat{u}_{1}^{(1)}+\widetilde{\mathbf{A}}_{p, 1} \widehat{\nabla} \widehat{u}_{2}^{(1)}\right)+\frac{\partial \psi}{\partial x_{3}}\right) \|_{L_{2}(\Omega)}^{2} .
\end{aligned}
$$


(iii) If the function $\kappa$ is also strictly positive, then we obtain an upper bound that does not contain the constants $C_{F, \Omega}$ and $C_{\Gamma, R}$ :

$$
\left\|u-\widehat{u}^{(1)}\right\| \leq \sqrt{M_{1}^{2}+\widetilde{M_{2}^{2}}+\widetilde{M}_{3 R}^{2}}+M_{3 \ominus \oplus}
$$

where $M_{1}$ and $\widetilde{M}_{2}^{2}$ are from (4.49) and (4.54), respectively, and

$$
\begin{aligned}
\widetilde{M}_{3 R}^{2}:=\left\|\kappa^{-1 / 2}\left(\kappa\left(\widehat{u}_{1}^{(1)}+x_{3} \widehat{u}_{2}^{(1)}\right)+\left(\widetilde{\mathbf{A}}_{p, 0} \widehat{\nabla} \widehat{u}_{1}^{(1)}+\widetilde{\mathbf{A}}_{p, 1} \widehat{\nabla} \widehat{u}_{2}^{(1)}, 0\right) \cdot \boldsymbol{\nu}_{R}\right)\right\|_{L_{2}\left(\Gamma_{R}\right)}^{2},(4.50 \\
M_{3 \oplus \ominus}:=c_{1}^{-1}\left(C_{\Gamma, \ominus}\left\|F_{\ominus}-\left(\widetilde{\mathbf{A}}_{p, 0} \widehat{\nabla} \widehat{u}_{1}^{(1)}+\widetilde{\mathbf{A}}_{p, 1} \widehat{\nabla} \widehat{u}_{2}^{(1)}, 0\right) \cdot \boldsymbol{\nu}_{\ominus}-\psi \nu_{\ominus 3}\right\|_{L_{2}\left(\Gamma_{\ominus}\right)}\right)^{(4.57} . \\
\left.\quad+C_{\Gamma, \oplus}\left\|F_{\oplus}-\left(\widetilde{\mathbf{A}}_{p, 0} \widehat{\nabla} \widehat{u}_{1}^{(1)}+\widetilde{\mathbf{A}}_{p, 1} \widehat{\nabla} \widehat{u}_{2}^{(1)}, 0\right) \cdot \boldsymbol{\nu}_{\oplus}-\psi \nu_{\oplus 3}\right\|_{L_{2}\left(\Gamma_{\oplus}\right)}\right) .
\end{aligned}
$$

Proof: We approximate the flux by

$$
\mathbf{y}=\left(\widetilde{\mathbf{A}}_{p, 0} \widehat{\nabla} \widehat{u}_{1}^{(1)}+\widetilde{\mathbf{A}}_{p, 1} \widehat{\nabla} \widehat{u}_{2}^{(1)}, 0\right)+\boldsymbol{\tau} \quad \text { with } \quad \boldsymbol{\tau}=\{0,0, \psi(x)\}^{T}
$$

and again verify that such $\mathbf{y}$ belongs to $H^{+}(\Omega$, Div). In (4.58) $\psi$ is again an auxiliary function from $L_{2}(\Omega)$ satisfying the conditions (4.52).

In order to rewrite estimate (4.15), we use (4.18)-(4.20) and obtain for the term $M_{1}$ with $v=\widehat{u}_{1}^{(1)}+x_{3} \widehat{u}_{2}^{(1)}$ and

$$
\nabla\left(x_{3} \widehat{u}_{2}^{(1)}\right)=\left(x_{3} \frac{\partial \widehat{u}_{2}^{(1)}}{\partial x_{1}}, x_{3} \frac{\partial \widehat{u}_{2}^{(1)}}{\partial x_{2}}, \widehat{u}_{2}^{(1)}\right)=\left(x_{3} \widehat{\nabla} \widehat{u}_{2}^{(1)}, \widehat{u}_{2}^{(1)}\right)
$$

the following representation:

$$
\begin{gathered}
M_{1}=\left(\int_{\Omega}\left(\left(\widehat{\nabla} \widehat{u}_{1}^{(1)}, 0\right)+\nabla\left(x_{3} \widehat{u}_{2}^{(1)}\right)-\mathbf{B y}\right) \cdot\left(\mathbf{A}\left(\left(\widehat{\nabla} \widehat{u}_{1}^{(1)}, 0\right)+\nabla\left(x_{3} \widehat{u}_{2}^{(1)}\right)\right)-\mathbf{y}\right) d x\right)^{1 / 2} \\
=\left(\int _ { \Omega } \left[\mathbf{A}\left(\left(\widehat{\nabla} \widehat{u}_{1}^{(1)}, 0\right)+\nabla\left(x_{3} \widehat{u}_{2}^{(1)}\right)\right) \cdot\left(\left(\widehat{\nabla} \widehat{u}_{1}^{(1)}, 0\right)+\nabla\left(x_{3} \widehat{u}_{2}^{(1)}\right)\right)\right.\right. \\
\left.\left.\quad-2 \mathbf{y} \cdot\left(\left(\widehat{\nabla} \widehat{u}_{1}^{(1)}, 0\right)+\nabla\left(x_{3} \widehat{u}_{2}^{(1)}\right)\right)+\mathbf{B y} \cdot \mathbf{y}\right] d x\right)^{1 / 2} .
\end{gathered}
$$

For the first term in (4.59), we have

$$
\begin{gathered}
\int_{\Omega} \mathbf{A}\left(\left(\widehat{\nabla} \widehat{u}_{1}^{(1)}, 0\right)+\right. \\
\left.\nabla\left(x_{3} \widehat{u}_{2}^{(1)}\right)\right) \cdot\left(\left(\widehat{\nabla} \widehat{u}_{1}^{(1)}, 0\right)+\nabla\left(x_{3} \widehat{u}_{2}^{(1)}\right)\right) d x=\int_{\widehat{\Omega}} d(\widehat{x})\left[\widetilde{\mathbf{A}}_{p, 0} \widehat{\nabla} \widehat{u}_{1}^{(1)} \cdot \widehat{\nabla} \widehat{u}_{1}^{(1)}\right. \\
\left.+2 \widetilde{\mathbf{A}}_{p, 1} \widehat{\nabla} \widehat{u}_{1}^{(1)} \cdot \widehat{\nabla} \widehat{u}_{2}^{(1)}+\widetilde{\mathbf{A}}_{p, 2} \widehat{\nabla} \widehat{u}_{2}^{(1)} \cdot \widehat{\nabla} \widehat{u}_{2}^{(1)}\right] d \widehat{x} \\
+\int_{\Omega} \widehat{u}_{2}^{(1)}\left(2 \mathbf{a}_{3} \cdot\left(\widehat{\nabla} \widehat{u}_{1}^{(1)}+x_{3} \widehat{\nabla} \widehat{u}_{2}^{(1)}, 0\right)+a_{33} \widehat{u}_{2}^{(1)}\right) d x
\end{gathered}
$$


Now we rewrite the second term in (4.59) in the form

$$
\begin{gathered}
\mathbf{y} \cdot\left(\left(\widehat{\nabla} \widehat{u}_{1}^{(1)}, 0\right)+\nabla\left(x_{3} \widehat{u}_{2}^{(1)}\right)\right)=\left(\left(\widetilde{\mathbf{A}}_{p, 0} \widehat{\nabla} \widehat{u}_{1}^{(1)}+\widetilde{\mathbf{A}}_{p, 1} \widehat{\nabla} \widehat{u}_{2}^{(1)}, 0\right)+\boldsymbol{\tau}\right) \cdot\left(\left(\widehat{\nabla} \widehat{u}_{1}^{(1)}, 0\right)+\nabla\left(x_{3} \widehat{u}_{2}^{(1)}\right)\right) \\
=\widetilde{\mathbf{A}}_{p, 0} \widehat{\nabla} \widehat{u}_{1}^{(1)} \cdot \widehat{\nabla} \widehat{u}_{1}^{(1)}+2 \widetilde{\mathbf{A}}_{p, 1} \widehat{\nabla} \widehat{u}_{2}^{(1)} \cdot \widehat{\nabla} \widehat{u}_{1}^{(1)}+x_{3} \widetilde{\mathbf{A}}{ }_{p, 1} \widehat{\nabla} \widehat{u}_{2}^{(1)} \cdot \widehat{\nabla} \widehat{u}_{2}^{(1)}+\widehat{u}_{2}^{(1)} \psi .
\end{gathered}
$$

Thus,

$$
\begin{aligned}
\int_{\Omega} \mathbf{y} \cdot\left(\left(\widehat{\nabla} \widehat{u}_{1}^{(1)}, 0\right)+\nabla\left(x_{3} \widehat{u}_{2}^{(1)}\right)\right) d x=\int_{\Omega} \widehat{u}_{2}^{(1)} \psi d x \\
\quad+\int_{\widehat{\Omega}} d(\widehat{x})\left(\widetilde{\mathbf{A}}_{p, 0} \widehat{\nabla} \widehat{u}_{1}^{(1)} \cdot \widehat{\nabla} \widehat{u}_{1}^{(1)}+2 \widetilde{\mathbf{A}}{ }_{p, 1} \widehat{\nabla} \widehat{u}_{2}^{(1)} \cdot \widehat{\nabla} \widehat{u}_{1}^{(1)}+\widetilde{\mathbf{A}}_{p, 2} \widehat{\nabla} \widehat{u}_{2}^{(1)} \cdot \widehat{\nabla} \widehat{u}_{2}^{(1)}\right) d \widehat{x}
\end{aligned}
$$

Finally, we note that

$$
\begin{aligned}
& \mathbf{B y} \cdot \mathbf{y}=\mathbf{B}\left(\left(\widetilde{\mathbf{A}}_{p, 0} \widehat{\nabla} \widehat{u}_{1}^{(1)}+\widetilde{\mathbf{A}}_{p, 1} \widehat{\nabla} \widehat{u}_{2}^{(1)}, 0\right)+\boldsymbol{\tau}\right) \cdot\left(\left(\widetilde{\mathbf{A}}_{p, 0} \widehat{\nabla} \widehat{u}_{1}^{(1)}+\widetilde{\mathbf{A}}_{p, 1} \widehat{\nabla} \widehat{u}_{2}^{(1)}, 0\right)+\boldsymbol{\tau}\right) \\
& =\mathbf{B}_{p, 0} \widetilde{\mathbf{A}}_{p, 0} \widehat{\nabla} \widehat{u}_{1}^{(1)} \cdot \widetilde{\mathbf{A}}_{p, 0} \widehat{\nabla} \widehat{u}_{1}^{(1)}+\mathbf{B}_{p, 0} \widetilde{\mathbf{A}}_{p, 0} \widehat{\nabla} \widehat{u}_{1}^{(1)} \cdot \widetilde{\mathbf{A}}_{p, 1} \widehat{\nabla} \widehat{u}_{2}^{(1)} \\
& +\mathbf{B}_{p, 0} \widetilde{\mathbf{A}}_{p, 1} \widehat{\nabla} \widehat{u}_{2}^{(1)} \cdot \widetilde{\mathbf{A}}_{p, 0} \widehat{\nabla} \widehat{u}_{1}^{(1)}+\mathbf{B}_{p, 0} \widetilde{\mathbf{A}}_{p, 1} \widehat{\nabla} \widehat{u}_{2}^{(1)} \cdot \widetilde{\mathbf{A}}_{p, 1} \widehat{\nabla} \widehat{u}_{2}^{(1)} \\
& +2\left(\mathbf{b}_{3} \cdot\left(\widetilde{\mathbf{A}}_{p, 0} \widehat{\nabla} \widehat{u}_{1}^{(1)}, 0\right)\right) \psi+2\left(\mathbf{b}_{3} \cdot\left(\widetilde{\mathbf{A}}_{p, 1} \widehat{\nabla} \widehat{u}_{2}^{(1)}, 0\right)\right) \psi+b_{33} \psi^{2},
\end{aligned}
$$

and, therefore,

$$
\begin{aligned}
\int_{\Omega} \mathbf{B y} \cdot \mathbf{y} d x & =\int_{\Omega}\left(b_{33} \psi^{2}+2\left(\mathbf{b}_{3} \cdot\left(\widetilde{\mathbf{A}}_{p, 0} \widehat{\nabla} \widehat{u}_{1}^{(1)}+\widetilde{\mathbf{A}}_{p, 1} \widehat{\nabla} \widehat{u}_{2}^{(1)}, 0\right)\right) \psi\right) d x \\
& +\int_{\widehat{\Omega}} d(\widehat{x}) \widetilde{\mathbf{B}}_{p, 0}\left(\widetilde{\mathbf{A}}_{p, 0} \widehat{\nabla} \widehat{u}_{1}^{(1)}+\widetilde{\mathbf{A}}_{p, 1} \widehat{\nabla} \widehat{u}_{2}^{(1)}, 0\right) \cdot\left(\widetilde{\mathbf{A}}_{p, 0} \widehat{\nabla} \widehat{u}_{1}^{(1)}+\widetilde{\mathbf{A}}_{p, 1} \widehat{\nabla} \widehat{u}_{2}^{(1)}, 0\right) d \widehat{x} .
\end{aligned}
$$

Substituting (4.60), (4.61) and (4.62) into (4.59), we obtain

$$
\begin{aligned}
M_{1}=\left(\int_{\Omega}\right. & {\left[\widehat{u}_{2}^{(1)}\left(2 \mathbf{a}_{3} \cdot\left(\widehat{\nabla} \widehat{u}_{1}^{(1)}+x_{3} \widehat{\nabla} \widehat{u}_{2}^{(1)}, 0\right)+a_{33} \widehat{u}_{2}^{(1)}-\psi\right)+b_{33} \psi^{2}\right.} \\
& \left.+2\left(\mathbf{b}_{3} \cdot\left(\widetilde{\mathbf{A}}_{p, 0} \widehat{\nabla} \widehat{u}_{1}^{(1)}+\widetilde{\mathbf{A}}_{p, 1} \widehat{\nabla} \widehat{u}_{2}^{(1)}, 0\right)\right) \psi\right] d x \\
+ & \int_{\widehat{\Omega}} d(\widehat{x})\left[\widetilde{\mathbf{B}}_{p, 0}\left(\left(\widetilde{\mathbf{A}}_{p, 0}-\widetilde{\mathbf{B}}_{p, 0}^{-1}\right) \widehat{\nabla} \widehat{u}_{1}^{(1)}+\widetilde{\mathbf{A}}_{p, 1} \widehat{\nabla} \widehat{u}_{2}^{(1)}\right) \cdot\left(\widetilde{\mathbf{A}}_{p, 0} \widehat{\nabla} \widehat{u}_{1}^{(1)}+\widetilde{\mathbf{A}}_{p, 1} \widehat{\nabla} \widehat{u}_{2}^{(1)}\right)\right. \\
& \left.\left.+\widehat{\nabla} \widehat{u}_{2}^{(1)} \cdot\left(\widetilde{\mathbf{A}}_{p, 1} \widehat{\nabla} \widehat{u}_{1}^{(1)}+\widetilde{\mathbf{A}}_{p, 2} \widehat{\nabla} \widehat{u}_{2}^{(1)}\right)\right] d \widehat{x}\right)^{1 / 2},
\end{aligned}
$$

To rearrange the functional $M_{2}$ for $\mathbf{y}$ from (4.58), we note that

$$
\operatorname{Div} \mathbf{y}=\operatorname{div} \widetilde{\mathbf{A}}_{p, 0} \widehat{\nabla} \widehat{u}_{1}^{(1)}+\operatorname{div} \widetilde{\mathbf{A}}_{p, 1} \widehat{\nabla} \widehat{u}_{2}^{(1)}+\frac{\partial \psi}{\partial x_{3}},
$$


and deduce from the product rule for the divergence and the first equation of (3.12)

$$
\begin{aligned}
\operatorname{div} \widetilde{\mathbf{A}}_{p, 0} \widehat{\nabla} \widehat{u}_{1}^{(1)}+\operatorname{div} \widetilde{\mathbf{A}}_{p, 1} \widehat{\nabla} \widehat{u}_{2}^{(1)}= & -\frac{\nabla d(\widehat{x})}{d(\widehat{x})} \cdot\left(\widetilde{\mathbf{A}}_{p, 0} \widehat{\nabla} \widehat{u}_{1}^{(1)}+\widetilde{\mathbf{A}}_{p, 1} \widehat{\nabla} \widehat{u}_{2}^{(1)}\right) \\
& +\left(\widetilde{\rho}_{0}^{2} \widehat{u}_{1}^{(1)}+\widetilde{\rho}_{1}^{2} \widehat{u}_{2}^{(1)}-\widehat{f}_{0}\right) .
\end{aligned}
$$

Hence, with the help of (3.3)-(3.5), we arrive at the relation

$$
\begin{aligned}
M_{2}=C_{F, \Omega} c_{1}^{-1} \|\left(f-\widehat{f}_{0}\right) & -\left(\rho^{2}-\widetilde{\rho}_{0}^{2}\right) \widehat{u}_{1}^{(1)}-\left(x_{3} \rho^{2}-\widetilde{\rho}_{1}^{2}\right) \widehat{u}_{2}^{(1)} \\
& -\frac{\nabla d(\widehat{x})}{d(\widehat{x})} \cdot\left(\widetilde{\mathbf{A}}_{p, 0} \widehat{\nabla} \widehat{u}_{1}^{(1)}+\widetilde{\mathbf{A}}_{p, 1} \widehat{\nabla} \widehat{u}_{2}^{(1)}\right)+\frac{\partial \psi}{\partial x_{3}} \|_{L_{2}(\Omega)} .
\end{aligned}
$$

We recall that the third component of the normal vectors $\boldsymbol{\nu}_{R}$ is zero. Then for $\mathbf{y}$ defined by (4.58), the functional $M_{3}$ has the form

$$
\begin{aligned}
M_{3} & :=c_{1}^{-1}\left(C_{\Gamma, R}\left\|\kappa\left(\widehat{u}_{1}^{(1)}+x_{3} \widehat{u}_{2}^{(1)}\right)+\left(\widetilde{\mathbf{A}}_{p, 0} \widehat{\nabla} \widehat{u}_{1}^{(1)}+\widetilde{\mathbf{A}}_{p, 1} \widehat{\nabla} \widehat{u}_{2}^{(1)}, 0\right) \cdot \boldsymbol{\nu}_{R}\right\|_{L_{2}\left(\Gamma_{R}\right)}\right. \\
& +C_{\Gamma, \ominus}\left\|F_{\ominus}-\left(\widetilde{\mathbf{A}}_{p, 0} \widehat{\nabla} \widehat{u}_{1}^{(1)}+\widetilde{\mathbf{A}}_{p, 1} \widehat{\nabla} \widehat{u}_{2}^{(1)}, 0\right) \cdot \boldsymbol{\nu}_{\ominus}-\psi \nu_{\ominus 3}\right\|_{L_{2}\left(\Gamma_{\ominus}\right)} \\
& \left.+C_{\Gamma, \oplus}\left\|F_{\oplus}-\left(\widetilde{\mathbf{A}}_{p, 0} \widehat{\nabla} \widehat{u}_{1}^{(1)}+\widetilde{\mathbf{A}}_{p, 1} \widehat{\nabla} \widehat{u}_{2}^{(1)}, 0\right) \cdot \boldsymbol{\nu}_{\oplus}-\psi \nu_{\oplus 3}\right\|_{L_{2}\left(\Gamma_{\oplus}\right)}\right) .
\end{aligned}
$$

(ii) The estimate (4.53) with $\widetilde{M}_{2}^{2}$ from (4.54) follows from Theorem 4.1 (ii) and (4.64) $-(4.65)$.

(iii) The statement follows from Theorem 4.1 (iii) and (4.67).

Remark 4.4 The function $\psi$ in (4.58) can be chosen using the same strategy (4.42), as in the case of the zero-order model, by setting

$$
\widehat{G}_{\oplus, \ominus}:=\widehat{F}_{\oplus, \ominus}-\left(\widetilde{\mathbf{A}}_{p, 0} \widehat{\nabla} \widehat{u}_{1}^{(1)}+\widetilde{\mathbf{A}}_{p, 1} \widehat{\nabla} \widehat{u}_{2}^{(1)}, 0\right) \cdot \boldsymbol{\nu}_{\oplus, \ominus}
$$

in (4.43).

\section{$5 \quad$ Particular cases}

Now we focus the attention on particular forms of the estimates (4.21) and (4.48) for domains with constant thickness and domains with plane parallel faces. In these cases, we have

$$
d_{\oplus}=d_{\ominus}+d_{0} \quad\left(d_{0}=\text { const }>0\right) .
$$

If we additionally assume that $\mathbf{A}=\mathbf{A}(\widehat{x})$ (this immediately implies $\mathbf{B}=\mathbf{B}(\widehat{x})$ ) and that

$$
a_{31}=a_{32}=0 \quad\left(\text { this yields } \mathbf{B}=\mathbf{A}^{-1}, b_{33}=a_{33}^{-1} b_{31}=b_{32}=0\right),
$$


and set $\psi$ as in (4.42), then the terms $M_{1}, M_{2}$ and $M_{3}$ become simpler. For the zero-order model and $\psi=\psi_{1}$ from (4.42), we have the estimate (4.21) with

$$
\begin{aligned}
M_{1} & =\left(\int_{\Omega} a_{33}^{-1} \psi_{1}^{2} d x\right)^{1 / 2} \\
& =\left(\int_{\widehat{\Omega}} a_{33}^{-1} \int_{d_{\ominus}}^{d_{\ominus}+d_{0}}\left(\widehat{\alpha}^{2} x_{3}^{2}+2 \widehat{\alpha} \widehat{\beta} x_{3}+\widehat{\beta}^{2}\right) d x_{3} d \widehat{x}\right)^{1 / 2} \\
& =\left(d_{0} \int_{\widehat{\Omega}} a_{33}^{-1}\left(\widehat{\alpha}^{2} \frac{d_{\oplus}^{2}+d_{\oplus} d_{\ominus}+d_{\ominus}^{2}}{3}+\widehat{\alpha} \widehat{\beta}\left(d_{\oplus}+d_{\ominus}\right)+\widehat{\beta}^{2}\right) d \widehat{x}\right)^{1 / 2}, \\
M_{2}=C_{F, \Omega} c_{1}^{-1}\left\|f-\widetilde{f}_{0}-\left(\rho^{2}-\widetilde{\rho}_{0}^{2}\right) \widehat{u}^{(0)}\right\|_{L_{2}(\Omega)}, &
\end{aligned}
$$

and

$$
M_{3}=C_{\Gamma, R} c_{1}^{-1}\left\|\kappa \widehat{u}^{(0)}+\left(\widetilde{\mathbf{A}}_{p, 0} \widehat{\nabla} \widehat{u}^{(0)}, 0\right) \cdot \boldsymbol{\nu}_{R}\right\|_{L_{2}\left(\Gamma_{R}\right)} .
$$

In the case of the first-order model, we use (4.58). Setting $\psi=\psi_{1}$, we obtain

$$
\begin{aligned}
M_{1}= & \left(\int_{\Omega}\left(a_{33}^{-1} \psi_{1}^{2}-2 \widehat{u}_{2}^{(1)} \psi_{1}+a_{33}\left(\widehat{u}_{2}^{(1)}\right)^{2}\right) d x\right)^{1 / 2} \\
= & \left(d_{0} \int_{\widehat{\Omega}} a_{33}^{-1}\left(\widehat{\alpha}^{2} \frac{d_{\oplus}^{2}+d_{\oplus} d_{\ominus}+d_{\ominus}^{2}}{3}+\widehat{\alpha} \widehat{\beta}\left(d_{\oplus}+d_{\ominus}\right)+\widehat{\beta}^{2}\right) d \widehat{x}\right. \\
& \left.-d_{0} \int_{\widehat{\Omega}} \widehat{u}_{2}^{(1)}\left(\widehat{\alpha}\left(d_{\oplus}+d_{\ominus}\right)+2 \widehat{\beta}-a_{33} \widehat{u}_{2}^{(1)}\right) d \widehat{x}\right)^{1 / 2}, \\
M_{2}= & C_{F, \Omega} c_{1}^{-1}\left\|f-\widetilde{f}_{0}-\left(\rho^{2}-\widetilde{\rho}_{0}^{2}\right) \widehat{u}_{1}^{(1)}-\left(x_{3} \rho^{2}-\widetilde{\rho}_{1}^{2}\right) \widehat{u}_{2}^{(1)}\right\|_{L_{2}(\Omega)},
\end{aligned}
$$

and

$$
M_{3}=C_{\Gamma} c_{1}^{-1}\left\|\kappa\left(\widehat{u}_{1}^{(1)}+x_{3} \widehat{u}_{2}^{(1)}\right)+\left(\widetilde{\mathbf{A}}_{p, 0} \widehat{\nabla} \widehat{u}_{1}^{(1)}+\widetilde{\mathbf{A}}_{p, 1} \widehat{\nabla} \widehat{u}_{2}^{(1)}, 0\right) \cdot \boldsymbol{\nu}_{R}\right\|_{L_{2}\left(\Gamma_{R}\right)} .
$$

We conclude from (5.3) and (5.6) that for the both models the term $M_{1}$ is of order $\mathcal{O}\left(d_{0}^{1 / 2}\right)$ when the plate thickness $d_{0}$ tends to zero. In the case of $f, \rho^{2} \in L^{\infty}(\Omega), M_{2}$ is for the both models also of the order $\mathcal{O}\left(d_{0}^{1 / 2}\right)$ (cf. (5.4) and (5.7)). 
For any domain with plane parallel faces, we can again make the assumption (5.2) but should strengthen the condition (5.1) replacing it by

$$
d_{\oplus}=\frac{d_{0}}{2}, d_{\ominus}=-\frac{d_{0}}{2} \quad\left(d_{0}=\text { const }>0\right) .
$$

Therefore, the function $\psi_{1}$ takes the simpler form

$$
\psi_{1}(x)=\frac{\widehat{F}_{\oplus}(\widehat{x})+\widehat{F}_{\ominus}(\widehat{x})}{d_{0}} x_{3}+\frac{\widehat{F}_{\oplus}(\widehat{x})-\widehat{F}_{\ominus}(\widehat{x})}{2},
$$

and the term $M_{1}$ in the case of the zero-order model, consequently, reduces to

$$
M_{1}=\left(\frac{d_{0}}{3} \int_{\widehat{\Omega}} a_{33}^{-1}\left(\widehat{F}_{\oplus}^{2}-\widehat{F}_{\oplus} \widehat{F}_{\ominus}+\widehat{F}_{\ominus}^{2}\right) d \widehat{x}\right)^{1 / 2} .
$$

For the first-order model, we have a more complicated form

$$
M_{1}=\left(\frac{d_{0}}{3} \int_{\widehat{\Omega}}\left(a_{33}^{-1}\left(\widehat{F}_{\oplus}^{2}-\widehat{F}_{\oplus} \widehat{F}_{\ominus}+\widehat{F}_{\ominus}^{2}\right)-3 \widehat{u}_{2}^{(1)}\left(\widehat{F}_{\oplus}-\widehat{F}_{\ominus}-a_{33} \widehat{u}_{2}^{(1)}\right)\right) d \widehat{x}\right)^{1 / 2} .
$$

\section{References}

[1] Alessandrini, A. L., Arnold, D. N., Falk, R. S., Madureira, A. L.: Derivation and justification of plate models by variational methods. In Plates and Shelles, Quebec 1996, (M. Fortin, ed.), pp. 1-20. CRM Proceedings and Lecture Notes, 21, American Mathematical Society, Providence, RI.

[2] Ainsworth, M. (1998): A posteriori error estimation for fully discrete hierarchic models of elliptic boundary value problems on thin domains. Numer. Math., 80, $325-362$.

[3] Babuška, I., Lee, I., Schwab, C. (1994): On the a posteriori estimation of the modeling error for the heat conduction in a plate and its use for adaptive hierarchical modeling. Appl. Numer. Math., 14, 5-21.

[4] Babuška, I., Schwab, C. (1996): A posteriori error estimation for hierarchic models of elliptic boundary value problems on thin domains. SIAM J. Numer. Anal., 33, $221-246$.

[5] Ciarlet, P.G.: Plates and junctions in elastic multi-structures. An asymptotic analysis. Recherches en Mathematiques Appliquees [Research in Applied Mathematics], 14. Masson, Paris; Springer-Verlag, Berlin, 1990.

[6] Ciarlet, P.G., Destuynder, P. (1979): A justification of a nonlinear model in plate theory. Comput. Methods Appl. Mech. Engrg., 17/18, 227258. 
[7] Ladevéze, P., Leguillon, D. (1983): Error estimate procedure in the finite element method and applications. SIAM J. Numer. Anal., 20, 485-509.

[8] Oden, J.T., Cho, J.R. (1996): Adaptive $h p q$-finite element methods of hierarchical models for plate- and shell-like structures. Comput. Meth. Appl. Mech. Engrg., 136, $317-345$.

[9] Repin, S.I. (2000): A posteriori error estimation for variational problems with uniformly convex functionals. Math. Comp., 69, 481-600.

[10] Repin, S.I. (2001): Estimates for errors in two-dimensional models of elasticity theory. J. Math. Sci, 106, pp. 3027-3041.

[11] Repin, S.I. (2008): A posteriori estimates for partial differential equations, Walter de Gryter, Berlin.

[12] Repin, S.I., Sauter, S.A. (2010): Computable estimates of the modeling error related to Kirchhoff-Love plate model. Anal. Appl., 8, 4, 409-428.

[13] Repin, S.I., Sauter, S.A., Smolianski, A.A. (2003): A posteriori error estimation for the Dirichlet problem with account of the error in the approximation of boundary conditions. Computing, 70, 205-233.

[14] Repin, S.I., Sauter, S.A., Smolianski, A.A. (2004): A posteriori error estimation for the Poisson equation with mixed Dirichlet/Neumann boundary conditions. J. Comput. Appl. Math., 164-165, 601-612.

[15] Repin, S.I., Sauter, S.A., Smolianski, A.A. (2004): A posteriori estimation of dimension reduction errors for elliptic problems in thin domains. SIAM J. Numer. Anal., 42, 4, 1435-1451.

[16] Stein, E., Ohnimus, S. (1997): Coupled model- and solution-adaptivity in the finiteelement method. Comput. Meth. Appl. Mech. Engrg., 150, 327-350.

[17] Synge, J.L.: The hypercircle in mathematical physics. Cambridge University Press, 1957.

[18] Vogelius, M., Babuška, I. (1981): On a dimensional reduction method I. The optimal selection of basis functions. Math. Comp., 37, 31-46.

[19] Vogelius, M., Babuška, I. (1981): On a dimensional reduction method III. A posteriori error estimation and an adaptive approach. Math. Comp., 37, 361-384. 